Kyushu J. Math.

Vol. 56, 2002, pp. 267-291

\title{
TUBES OF NON-CONSTANT RADIUS IN SYMMETRIC SPACES
}

\author{
Naoyuki KOIKE \\ (Received 26 January 2001)
}

\section{Introduction}

A hypersurface can often be caught as a tube over its focal submanifold. Conversely, from a given submanifold, a family of hypersurfaces are constructed by considering tubes of various radii. Hence the notion of a tube is important in the study of hypersurfaces. This notion is used to characterize various classes of hypersurfaces (for example, isoparametric hypersurfaces in real space form and certain types of real hypersurfaces in a complex space form). In almost every characterization, tubes of constant radius are used. However, tubes of non-constant radius should also be used to characterize comparatively large classes of hypersurfaces. In fact, for example, there exist examples of a Dupin hypersurface (or more generally, the curvature netted hypersurface defined in [11]) which are caught as a tube of non-constant radius. We also note that rotational hypersurfaces (other than cylinders) in a Euclidean space are caught as tubes of non-constant radius over a geodesic.

In [14], Reckziegel investigated the shape operator of a tube of constant radius in real space form. See [7] for its investigation in a general Riemannian manifold. In [10], we investigated the shape operator of a tube of non-constant radius in a Euclidean space. In this paper, we shall describe the shape operator of a tube of non-constant radius over a certain type of submanifold in a symmetric space. These descriptions will be useful in finding the conditions for which the tube will satisfy various geometric properties. In fact, in terms of the description, we shall find the minimality conditions for tubes over a geodesic in real space form and an invariant geodesic in a simply connected rank-one symmetric space (other than a real space form). Most tubes with these conditions become inhomogeneous minimal hypersurfaces.

Now we shall prepare to state the results in this paper. Let $N=G / K$ be a symmetric space and $(\mathfrak{g}, \tau)$ be the orthogonal symmetric Lie algebra associated with $N$. Let $\mathfrak{p}=\{X \in \mathfrak{g} \mid \tau(X)=-X\}$. The subspace $\mathfrak{p}$ is identified with the tangent space $T_{e K} G / K$ of $G / K$ at $e K$, where $e$ is the identity element of $G$. For $w \in T_{g_{K}} N$, 
we define linear transformations $D_{w}^{c o}$ and $D_{w}^{s i}$ of $T_{g K} N$ by

$$
D_{w}^{c o}=g_{*} \cos \left(\sqrt{-1} \operatorname{ad}\left(g_{*}^{-1} w\right)\right) g_{*}^{-1}, \quad D_{w}^{s i}=g_{*} \frac{\sin \left(\sqrt{-1} \operatorname{ad}\left(g_{*}^{-1} w\right)\right)}{\sqrt{-1} \operatorname{ad}\left(g_{*}^{-1} w\right)} g_{*}^{-1},
$$

respectively, where ad is the adjoint representation of $\mathfrak{g}$ and $g_{*}$ is the differential of $g$. Let $(M, f)$ be an $n$-dimensional submanifold in $N$ (i.e. $f$ is an isometric immersion of $M$ into $N)$ and $\left(t_{\varepsilon}(M), f_{\varepsilon}\right)$ be the $\varepsilon$-tube over $(M, f)$, where $\varepsilon$ may not be constant. Denote by $\pi_{\varepsilon}$ the natural projection of $t_{\varepsilon}(M)$ onto $M$, by $\operatorname{pr}_{\varepsilon}^{T}$ the orthogonal projection of $f_{\varepsilon}^{*} T N$ onto $T t_{\varepsilon}(M)$ and by $P_{\gamma_{\xi}}\left(\xi \in T^{\perp} M\right)$ the parallel translation along a geodesic $\gamma_{\xi}$ in $N$ with $\dot{\gamma}_{\xi}(0)=\xi$ from $\gamma_{\xi}(0)$ to $\gamma_{\xi}(1)$, where $\dot{\gamma}_{\xi}(0)$ is the velocity vector of $\gamma_{\xi}$ at 0 . In the following, we shall suppress $f_{*}$ and $f_{\varepsilon *}$ unless necessary. In the case where $(M, f)$ has a root decomposable normal bundle (see Section 2 for a definition), for $\xi \in T_{x}^{\perp} M$, we can define a linear transformation $B_{\xi}$ of $T_{x} M$ by

$$
B_{\xi} X=D_{\xi}^{c o} X-D_{\xi}^{s i} A_{\xi} X \quad\left(X \in T_{x} M\right)
$$

In the case where $(M, f)$ has a Lie triple systematic and root decomposable normal bundle (see Section 2 for this definition), we can obtain the following descriptions for the shape operator of $\left(t_{\varepsilon}(M), f_{\varepsilon}\right)$.

THEOREM A. Let $(M, f)$ be a submanifold with a Lie triple systematic and root decomposable normal bundle in a symmetric space $N=G / K$. Denote by $A$ (respectively $A^{\varepsilon}$ ) the shape tensor of $(M, f)$ (respectively $\left(t_{\varepsilon}(M), f_{\varepsilon}\right)$ ) and by $E$ the inward unit normal vector field of $\left(t_{\varepsilon}(M), f_{\varepsilon}\right)$. Then we have the following statements (i) and (ii).

(i) Let $v$ be a vertical vector of $t_{\varepsilon}(M)$ at $\xi \in \pi_{\varepsilon}^{-1}(x)$ (i.e. $v \in T_{\xi} \pi_{\varepsilon}^{-1}(x)$ ) such that $g_{*}^{-1} P_{\gamma_{\xi}}^{-1} v$ is a root vector for a root $\mu$, where $f(x)=g K$ and we fix a maximal abelian subspace in $\mathfrak{p}$ containing $g_{*}^{-1} \xi$. Then we have

$$
A_{E(\xi)}^{\varepsilon} v=\frac{1}{\sqrt{1+\left\|B_{\xi}^{-1} \operatorname{grad} \varepsilon\right\|^{2}}}\left\{\frac{\mu\left(g_{*}^{-1} \xi\right)}{\varepsilon(x) \tan \mu\left(g_{*}^{-1} \xi\right)} v-\operatorname{pr}_{\varepsilon}^{T} P_{\gamma_{\xi}} g_{*} Z_{v}(1)\right\}
$$

where $Z_{v}$ is the curve in $\mathfrak{p}$ satisfying (2.2) and (2.3).

(ii) Let $X$ be a tangent vector of $M$ at $x$ such that $A_{\xi} X=\lambda X$ and $g_{*}^{-1} X$ is a root vector for a root $\mu$, where $f(x)=g K$ and we fix a maximal abelian subspace in $\mathfrak{p}$ 


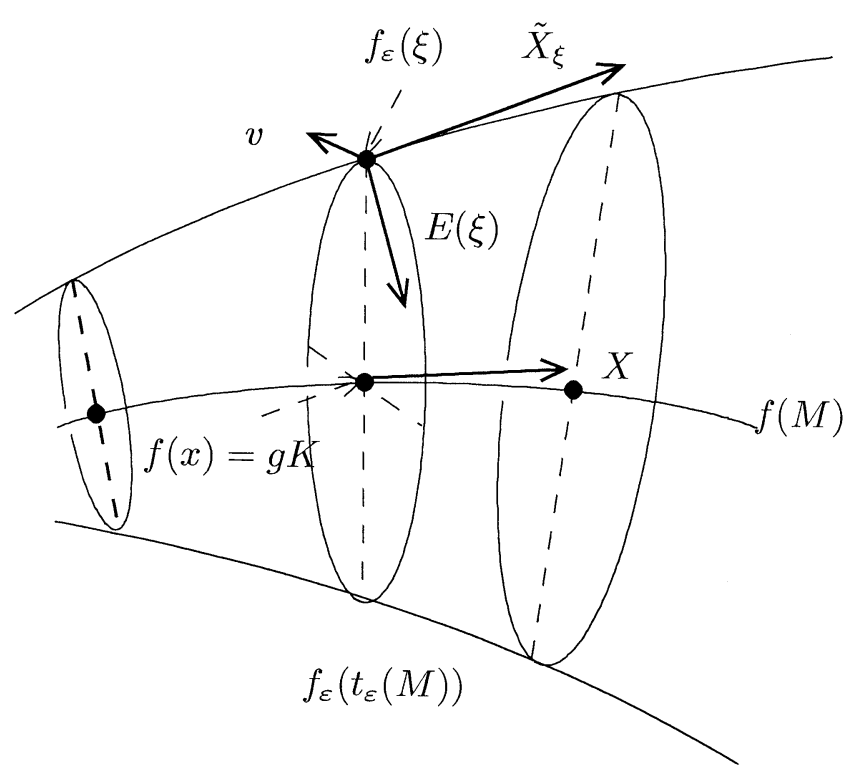

FIGURE 1.

containing $g_{*}^{-1} \xi$. Then, for the natural lift $\tilde{X}_{\xi}$ of $X$ to $\xi\left(\in \pi_{\varepsilon}^{-1}(x)\right)$, we have

$$
\begin{aligned}
A_{E(\xi)}^{\varepsilon} \tilde{X}_{\xi}= & \frac{1}{\sqrt{1+\left\|B_{\xi}^{-1} \operatorname{grad} \varepsilon\right\|^{2}}}\left\{\frac{\lambda+\mu\left(g_{*}^{-1} \xi\right) \tan \mu\left(g_{*}^{-1} \xi\right)}{\varepsilon(x)\left(\lambda \tan \mu\left(g_{*}^{-1} \xi\right) / \mu\left(g_{*}^{-1} \xi\right)-1\right)}\right. \\
& \left.\times\left(\tilde{X}_{\xi}-\frac{X \varepsilon}{1+\left\|B_{\xi}^{-1} \operatorname{grad} \varepsilon\right\|^{2}} B_{\xi}^{-2} \operatorname{grad} \varepsilon_{\xi}\right)-\operatorname{pr}_{\varepsilon}^{T} P_{\gamma_{\xi}} g_{*} Z_{X, \xi}(1)\right\},
\end{aligned}
$$

where $Z_{X, \xi}$ is the curve in $\mathfrak{p}$ satisfying (2.4) and (2.5).

\section{Remark 0.1.}

(i) If $\mu\left(g_{*}^{-1} \xi\right)=0$, then $\mu\left(g_{*}^{-1} \xi\right) / \tan \mu\left(g_{*}^{-1} \xi\right)$ implies 1 .

(ii) If $\varepsilon$ is constant, then the assumption that $(M, f)$ has Lie triple systematic normal bundle is not necessary in Theorem A.

(iii) If $\varepsilon$ is constant, then $Z_{v}$ and $Z_{X, \xi}$ vanish. 
(iv) All hypersurfaces in a general symmetric space, all submanifolds in a real space form, all invariant (i.e. Kaehlerian) submanifolds and all generic (in particular, Lagrangian) ones in a complex space form, all invariant submanifolds (which are automatically totally geodesic) in other rank-one symmetric space and all halfdimensional totally complex submanifolds in a quaternionic space form have a Lie triple systematic and root decomposable normal bundle (see Proposition 2.2).

(v) All submanifolds with an abelian normal bundle in a general symmetric space (in particular, principal orbits of the isotropy representation of a general symmetric space) have Lie triple systematic and root decomposable normal bundles.

(vi) All curvature adapted submanifolds in general symmetric space have root decomposable normal bundles.

(vii) If submanifolds $\left(M_{i}, f_{i}\right)$ in a symmetric space $N_{i}(i=1, \ldots, k)$ have Lie triple systematic (respectively root decomposable) normal bundles, then the product submanifold $\left(M_{1} \times \cdots \times M_{k}, f_{1} \times \cdots \times f_{k}\right)$ in the product symmetric space $N_{1} \times \cdots \times N_{k}$ also has a Lie triple systematic (respectively root decomposable) normal bundle.

(viii) In the case where the ambient space is a rank-one symmetric space or $(M, f)$ is a principal orbit of the isotropy representation of a general symmetric space, the curves $Z_{v}$ and $Z_{X, \xi}$ can be concretely described (see Sections 4-6).

Denote by $N^{m}(c)$ the $m$-dimensional real space form of constant curvature $c$, by $\mathbf{C} N^{m}(c)$ (respectively $\mathbf{Q} N^{m}(c)$ ) the $m$-dimensional complex (respectively quaternionic) space form of constant holomorphic (respectively quaternionic) sectional curvature $c$ and by $\mathbf{O} N^{2}(c)$ the Cayley projective (or Cayley hyperbolic) plane of constant Cayley sectional curvature $c$. In terms of the descriptions of Theorem A, we can find the minimality conditions for tubes over a geodesic in $N^{m}(c)$ and an invariant geodesic in $\mathbf{F} N^{m}(c)(\mathbf{F}=\mathbf{C}, \mathbf{Q}$ or $\mathbf{O}, m=2$ when $\mathbf{F}=\mathbf{O})$.

THEOREM B. Let $\gamma$ be a geodesic in the $m$-dimensional real space form $N^{m}(c)$ of constant curvature $c$. The $\varepsilon$-tube $t_{\varepsilon}(\gamma)$ is minimal if and only if $\varepsilon$ satisfies the differential equation

$$
\begin{aligned}
\varepsilon^{\prime \prime}= & -\left\{\sqrt{c} \tan (\sqrt{c} \varepsilon)+\frac{2 c \varepsilon}{\cos ^{2}(\sqrt{c} \varepsilon)}-\frac{(m-2) \sqrt{c}}{\tan (\sqrt{c} \varepsilon)}\right\} \varepsilon^{\prime 2} \\
& -\sqrt{c} \tan (\sqrt{c} \varepsilon) \varepsilon^{\prime}-\frac{\sqrt{c}}{2} \sin (2 \sqrt{c} \varepsilon)+\frac{(m-2) \sqrt{c} \cos ^{2}(\sqrt{c} \varepsilon)}{\tan (\sqrt{c} \varepsilon)}
\end{aligned}
$$

where $\varepsilon^{\prime}=d \varepsilon / d s$ and $\varepsilon^{\prime \prime}=d^{2} \varepsilon / d s^{2}(s$ is the arclength of $\gamma)$. 


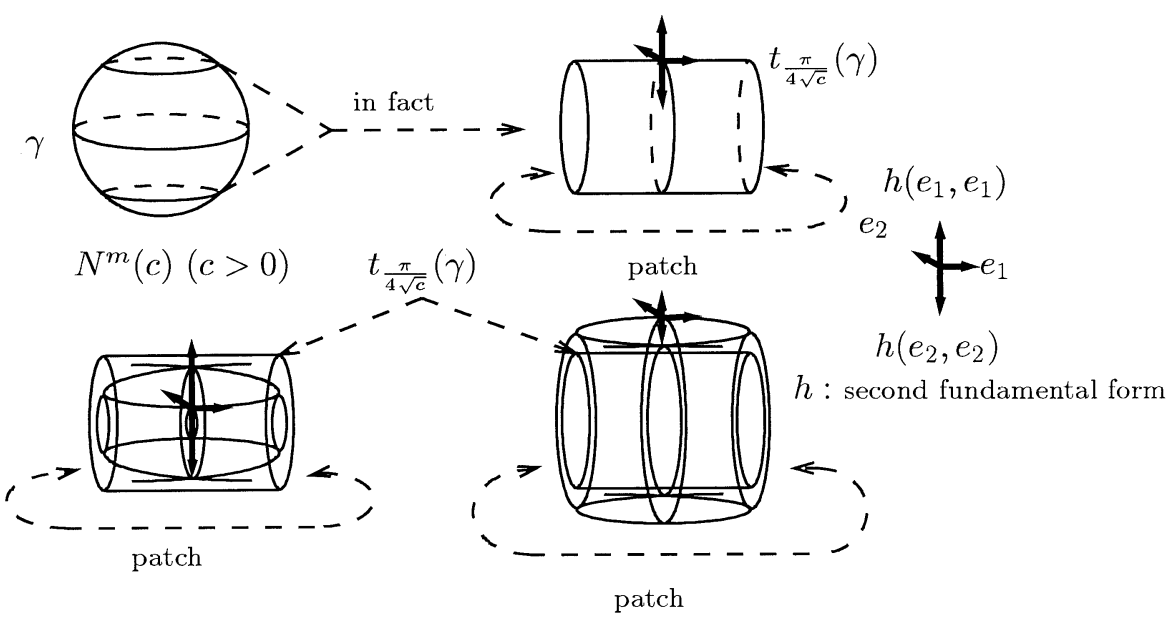

FIGURE 2.

\section{Remark 0.2.}

(i) The condition for $t_{\varepsilon}(\gamma)$ to have constant mean curvature is generally fulfilled (see the proof of Theorem B in detail).

(ii) In general, we can fulfil the condition for an $\varepsilon$-tube over a general dimensional totally geodesic submanifold in $N^{m}(c)$ to be minimal.

(iii) According to this theorem, we can obtain a two-parameter (one-parameter up to congruence) family of inhomogeneous minimal hypersurfaces in a real space form.

(iv) If $c=0$ and $m=3$, then this differential equation becomes $\varepsilon \varepsilon^{\prime \prime}=\varepsilon^{\prime 2}+1$ and hence $\varepsilon=(1 / a) \cosh (a s+b)(a, b \in \mathbf{R})$, that is, $t_{\varepsilon}(\gamma)$ implies the catenoid. Thus, for a positive function $\varepsilon$ satisfying the differential equation in Theorem $\mathrm{B}$, the $\varepsilon$-tube $t_{\varepsilon}(\gamma)$ is interpreted as a minimal hypersurface of catenoid type in $N^{m}(c)$.

(v) If $c>0, m=3$ and $\varepsilon$ is constant, then the differential equation in Theorem $\mathrm{B}$ becomes $\cos (\sqrt{c} \varepsilon) \cos (2 \sqrt{c} \varepsilon)=0$. Hence we have $\varepsilon=\pi / 4 \sqrt{c}$ because the focal radius of $\gamma$ is $\pi / 2 \sqrt{c}$. That is, $t_{\varepsilon}(\gamma)$ implies the minimal Clifford torus. If $c>0$, $m=3$ and $\varepsilon$ is non-constant, then the minimal $\varepsilon$-tube in Theorem B distributes inside or outside the minimal Clifford torus $t_{\pi / 4 \sqrt{c}}(\gamma)$ (see Figure 2).

Let $\gamma$ be an immersion of a domain $D$ of $\mathbf{F}$ into $\mathbf{F} N^{m}(c)$. If $(D, \gamma)$ is a totally geodesic invariant submanifold with respect to the induced metric on $D$, then we shall call $\gamma$ an invariant geodesic. In terms of the descriptions of Theorem A, we can find the minimality conditions of tubes over an invariant geodesic. 
THEOREM C. Let $\gamma$ be an invariant geodesic in the $m$-dimensional rank-one symmetric space $\mathbf{F} N^{m}(c)$, where $\mathbf{F}=\mathbf{C}, \mathbf{Q}$ or $\mathbf{O}$ and $m=2$ when $\mathbf{F}=\mathbf{O}$. The $\varepsilon$-tube $t_{\varepsilon}(\gamma)$ is minimal if and only if $\varepsilon$ satisfies the partial differential equation

$$
\begin{aligned}
\sum_{i=0}^{q-1} \sum_{j=0}^{q-1} \varepsilon_{x_{i}} \varepsilon_{x_{j}} \varepsilon_{x_{i} x_{j}}-\rho^{2}\left(\cos ^{2} \frac{\sqrt{c} \varepsilon}{2}+\sum_{i=0}^{q-1} \varepsilon_{x_{i}}^{2}\right) \sum_{j=0}^{q-1} \varepsilon_{x_{j} x_{j}} \\
=\rho^{4} \cos ^{2} \frac{\sqrt{c} \varepsilon}{2}\left(\frac{q \sqrt{c}}{2} \tan \frac{\sqrt{c} \varepsilon}{2}-\frac{(m-2) q \sqrt{c}}{2 \tan \frac{1}{2} \sqrt{c} \varepsilon}-\frac{(q-1) \sqrt{c}}{\tan (\sqrt{c} \varepsilon)}\right) \\
\quad \times\left(\rho^{2} \cos ^{2} \frac{\sqrt{c} \varepsilon}{2}+\sum_{i=0}^{q-1} \varepsilon_{x_{i}}^{2}\right) \\
\quad-\rho^{2}\left(\frac{\sqrt{c}}{2} \sin (\sqrt{c} \varepsilon)+\sqrt{c} \cos \frac{\sqrt{c} \varepsilon}{2}\right) \sum_{i=0}^{q-1} \varepsilon_{x_{i}}^{2}+\sum_{i=0}^{q-1} \sum_{j=0}^{q-1} \sum_{k=0}^{q-1} \Gamma_{i j}^{k} \varepsilon_{x_{i}} \varepsilon_{x_{j}} \varepsilon_{x_{k}} \\
\quad-\left(\rho^{2} \cos ^{2} \frac{\sqrt{c} \varepsilon}{2}+\sum_{i=0}^{q-1} \varepsilon_{x_{i}}^{2}\right) \sum_{j=0}^{q-1} \sum_{k=0}^{q-1} \Gamma_{j j}^{k} \varepsilon_{x_{k}} .
\end{aligned}
$$

Here $q=2,4$ or 8 depending on whether $\mathbf{F}=\mathbf{C}, \mathbf{Q}$ or $\mathbf{O},\left(x_{0}, \ldots, x_{q-1}\right)$ is the coordinate of $\mathbf{F}$ given by $z=x_{0} \cdot 1+\sum_{i=1}^{q-1} x_{i} e_{i}(z \in \mathbf{F})$, where $1, e_{1}, \ldots, e_{q-1}$ is the usually ordered generator of $\mathbf{F}$,

$$
\begin{gathered}
\varepsilon_{x_{i}}=\frac{\partial \varepsilon}{\partial x_{i}}, \quad \varepsilon_{x_{i} x_{j}}=\frac{\partial^{2} \varepsilon}{\partial x_{i} \partial x_{j}} \quad(i, j=0, \ldots, q-1), \\
\rho:=\left\langle\gamma_{*} \frac{\partial}{\partial x_{i}}, \gamma_{*} \frac{\partial}{\partial x_{i}}\right\rangle \quad(i=0, \ldots, q-1)
\end{gathered}
$$

and

$$
\nabla_{\partial / \partial x_{i}} \frac{\partial}{\partial x_{j}}=\sum_{k=0}^{q-1} \Gamma_{i j}^{k} \frac{\partial}{\partial x_{k}},
$$

where $\nabla$ is the Levi-Civita connection of the induced metric on the domain of $\gamma$.

Remark 0.3.

(i) The previous partial differential equation is quasi-linear and hence it is integrable, where we note that $\rho$ and $\Gamma_{i j}^{k}$ are of class $C^{\omega}$. Therefore, we can obtain a family of non-homogeneous minimal real hypersurfaces in $\mathbf{F} N^{m}(c)$.

(ii) The condition for $t_{\varepsilon}(\gamma)$ to have constant mean curvature is generally fulfilled (see the proof of Theorem $\mathrm{C}$ in detail). 
(iii) In general, we can find the condition for an $\varepsilon$-tube over a general dimensional totally geodesic invariant submanifold in $\mathbf{F} N^{m}(c)$ to be minimal.

In Section 1, we shall recall basic notions and facts. In Section 2, we define two new classes of submanifolds in a symmetric space and prove some facts for these new classes. In Section 3, we shall prove Theorem A. Also, in Sections 4 and 5, we shall prove Theorems B and C, respectively. In Section 6, we shall investigate the $\varepsilon$-tube over the principal orbit of the isotropy representation of a symmetric space.

Throughout this paper, unless otherwise mentioned, we assume that all geometric objects are of class $C^{\infty}$ and all manifolds are connected ones without boundary.

\section{Basic notions and facts}

In this section, we shall recall basic notions and facts. Let $f$ be an isometric immersion of a Riemannian manifold $M$ into a Riemannian manifold $\tilde{M}$. Then we call $(M, f)$ a submanifold in $\tilde{M}$. For simplicity, in the case where $M \subset \tilde{M}$ and $f$ is the inclusion map, we shall simply write the submanifold $(M, f)$ as $M$. Denote by $T^{\perp} M$ the normal bundle of $(M, f)$ and by $\pi$ the bundle projection of $T^{\perp} M$. Let $U$ be the domain of the normal exponential map $\exp ^{\perp}$ of $(M, f)$. For a positive function $\varepsilon$ on $M$, set $B_{\varepsilon}(M):=\left\{\xi \in T^{\perp} M \mid\|\xi\| \leq \varepsilon(\pi(\xi))\right\}$ and $t_{\varepsilon}(M):=\partial B_{\varepsilon}(M)$, where $\|\xi\|$ is the norm of $\xi$ and $\partial B_{\varepsilon}(M)$ is the boundary of $B_{\varepsilon}(M)$. Set $\pi_{\varepsilon}:=\left.\pi\right|_{t_{\varepsilon}}(M)$. If $t_{\varepsilon}(M) \subset U$, then we set $f_{\varepsilon}:=\left.\exp ^{\perp}\right|_{t_{\varepsilon}(M)}$. Denote by $F(M, f)$ the focal set of $(M, f)$. If $\varepsilon$ satisfies $\exp ^{\perp}\left(B_{\varepsilon}(M)\right) \cap F(M, f)=\emptyset$, then $\left(t_{\varepsilon}(M), f_{\varepsilon}\right)$ becomes a hypersurface in $\tilde{M}$. This hypersurface is called the tube of radius $\varepsilon$ over $(M, f)$. In the following, we shall call it the $\varepsilon$-tube for simplicity. Note that $\left(t_{\varepsilon}(M), f_{\varepsilon}\right)$ implies a geodesic sphere of radius $\varepsilon$ in the case where $(M, f)$ is one point and that it implies a hypersurface parallel to $(M, f)$ in the case where $(M, f)$ is a hypersurface and $\varepsilon$ is constant. Now we shall recall the notion of a natural lift defined in [10]. Denote by $\nabla$ (respectively $\tilde{\nabla}$ ) the Levi-Civita connection of $M$ (respectively $\tilde{M}$ ) and by $\nabla^{\perp}$ the normal connection of $(M, f)$. Also, denote by $A$ and $A^{\varepsilon}$ the shape tensors of $(M, f)$ and $\left(t_{\varepsilon}(M), f_{\varepsilon}\right)$, respectively. Let $H$ be the horizontal distribution on $T^{\perp} M$ associated with $\nabla^{\perp}$ and by $X_{\xi}^{L}$ the horizontal lift of $X \in T_{x} M$ to $\xi\left(\in \pi^{-1}(x)=T_{x}^{\perp} M\right)$ with respect to $H$. For $X \in T_{x} M$ and $\xi \in \pi_{\varepsilon}^{-1}(x)$, it is shown that $X_{\xi}^{L}+(X \varepsilon / \varepsilon(x)) \xi$ $\left(\in T_{\xi}\left(T^{\perp} M\right)\right.$ ) is tangent to $t_{\varepsilon}(M)$ (see Lemma 1.1 of [10]), where $\xi$ is regarded as a vector of $T_{\xi}\left(T^{\perp} M\right)$ under the natural identification of $T_{x}^{\perp} M$ and $T_{\xi}\left(T_{x}^{\perp} M\right)$. This vector $X_{\xi}^{L}+(X \varepsilon / \varepsilon(x)) \xi$ is called the natural lift of $X$ to $\xi$ and is denoted by $\tilde{X}_{\xi}$. The vertical distribution $V^{\varepsilon}$ and the horizontal distribution $H^{\varepsilon}$ on $t_{\varepsilon}(M)$ are defined 
by $V_{\xi}^{\varepsilon}=T_{\xi}\left(\pi_{\varepsilon}^{-1}\left(\pi_{\varepsilon}(\xi)\right)\right)\left(\xi \in t_{\varepsilon}(M)\right)$ and $H_{\xi}^{\varepsilon}=\left\{\tilde{X}_{\xi} \mid X \in T_{\pi_{\varepsilon}(\xi)} M\right\}\left(\xi \in t_{\varepsilon}(M)\right)$, respectively. For $\xi \in T_{x}^{\perp} M$ and $X \in T_{x} M$, denote by $J_{\xi, X}$ the Jacobi field along $\gamma_{\xi}$ with $J_{\xi, X}(0)=X$ and $J_{\xi, X}^{\prime}(0)=-A_{\xi} X$, where $\gamma_{\xi}$ is the geodesic in $\tilde{M}$ with $\dot{\gamma}_{\xi}(0)=\xi$ and $J_{\xi, X}^{\prime}=\tilde{\nabla}_{\dot{\gamma}_{\xi}} J_{\xi, X}$. It is clear that $\exp _{*}^{\perp}\left(X_{\xi}^{L}\right)=J_{\xi, X}(1)$ holds. Hence we have

$$
f_{\varepsilon *} \tilde{X}_{\xi}=J_{\xi, X}(1)+\frac{X \varepsilon}{\varepsilon(x)} \dot{\gamma}_{\xi}(1) .
$$

Next we shall recall basic notions and facts with respect to a symmetric space. Let $N=G / K$ be a symmetric space and $(\mathfrak{g}, \tau)$ be the orthogonal symmetric Lie algebra associated with $N$ and $\mathfrak{p}=\{X \in \mathfrak{g} \mid \tau(X)=-X\}$. Let $\triangle$ be the root system with respect to a maximal abelian subspace $\mathfrak{h}$ in $\mathfrak{p}$. If $N$ is compact (respectively non-compact), then each root is a real (respectively purely imaginary) valued form on $\mathfrak{h}$ and that if $N$ is of Euclidean type, then $\mathfrak{h}=\mathfrak{p}$ and $\Delta=\emptyset$. The subspace $\mathfrak{p}_{\mu}=\left\{X \in \mathfrak{p} \mid \operatorname{ad}(Y)^{2}(X)=-\mu(Y)^{2} X\right.$ for all $\left.Y \in \mathfrak{h}\right\}(\mu \in \Delta)$ is called the root space for $\mu$. Here we note that, in the case where $N$ is non-compact, these roots imply $\sqrt{-1}$ multiples of the usual roots. We shall call elements of $\mathfrak{p}_{\mu}$ root vectors for $\mu$ and interpret $\mathfrak{h}$ as the root space for 0 . Let $\Delta_{+}$be the set of all positive roots with respect to some lexicographic ordering of $\mathfrak{h}$. Then we have $\mathfrak{p}=\mathfrak{h}+\sum_{\mu \in \Delta_{+}} \mathfrak{p}_{\mu}$, which is called the root space decomposition with respect to $\mathfrak{h}$. A Jacobi field $J$ along a geodesic $\gamma$ in $N$ is described as

$$
J(s)=P_{\gamma \mid[0, s]}\left(D_{s \dot{\gamma}(0)}^{c o} J(0)+s \cdot D_{s \dot{\gamma}(0)}^{s i} J^{\prime}(0)\right),
$$

where $D_{s g_{*}^{-1} \dot{\gamma}(0)}^{c o}$ and $D_{s g_{*}^{-1} \dot{\gamma}(0)}^{s i}$ are the linear transformations of $\mathfrak{p}$ stated in Introduction and $J^{\prime}(0)=\tilde{\nabla}_{\dot{\gamma}(0)} J(\tilde{\nabla}$ is the Levi-Civita connection of $N)$.

\section{Two new classes of submanifolds in a symmetric space}

Let $(M, f)$ be a submanifold in a symmetric space $N=G / K$. If, for each $x \in M$, $g_{*}^{-1}\left(T_{x}^{\perp} M\right)(f(x)=g K)$ is abelian in $\mathfrak{p}$, then $(M, f)$ is said to have an abelian normal bundle. In this section, we shall define two new classes of submanifolds in a symmetric space. If, for each $x \in M(f(x)=g K), g_{*}^{-1}\left(T_{x}^{\perp} M\right)$ is a Lie triple system in $\mathfrak{p}$, then we shall say that $(M, f)$ has a Lie triple systematic normal bundle. Also, if, for each $\xi(\neq 0) \in T^{\perp} M$, there exists a maximal abelian subspace $\mathfrak{h}$ in $\mathfrak{p}$ containing $g_{*}^{-1} \xi$ such that $g_{*}^{-1}\left(T_{x}^{\perp} M\right)=\mathfrak{h} \cap g_{*}^{-1}\left(T_{x}^{\perp} M\right)+\sum_{\mu \in \Delta_{+}}\left(\mathfrak{p}_{\mu} \cap g_{*}^{-1}\left(T_{x}^{\perp} M\right)\right)$ ( $x$ is the base point of $\xi, f(x)=g K$ ), then we shall say that $(M, f)$ has a root decomposable normal bundle, where $\Delta_{+}$is the positive root system with respect to 
$\mathfrak{h}$ and $\mathfrak{p}_{\mu}\left(\mu \in \Delta_{+}\right)$is the root space for $\mu$. In [1], Berndt and Vanhecke called $(M, f)$ a curvature adapted submanifold if, for each $v \in T^{\perp} M$, the operator $R(\cdot, v) v$ leaves $T_{x} M$ invariant ( $x$ is the base point of $v$ ) and it is commutative with the shape operator $A_{v}$. According to the following lemma, curvature adapted submanifolds have root decomposable normal bundles.

LEMMA 2.1. A submanifold $(M, f)$ has a root decomposable normal bundle if and only if, for each $v \in T^{\perp} M$, the operator $R(\cdot, v) v$ leaves $T_{x} M$ invariant ( $x$ is the base point of $v$ ).

Proof. Let $f(x)=g K$. It is clear that the operator $R(\cdot, v) v$ leaves $T_{x} M$ invariant if and only if it leaves $T_{x}^{\perp} M$ invariant. Also, since $g_{*}^{-1} R(\cdot, v) v g_{*}=-\operatorname{ad}(v)^{2}$, the operator $R(\cdot, v) v$ leaves $T_{x}^{\perp} M$ invariant if and only if $g_{*}^{-1} T_{x}^{\perp} M$ has a frame consisting of root vectors. Thus the statement is proved.

Now we shall give examples of submanifolds belonging to these new classes. Let $\phi_{1}$ be the complex structure of the $m$-dimensional complex space form $\mathbf{C} N^{m}(c)$, $\left\{\phi_{1}, \phi_{2}, \phi_{3}\right\}$ be the quaternionic structure of the $m$-dimensional quaternionic space form $\mathbf{Q} N^{m}(c)$ and $\left\{\phi_{1}, \ldots, \phi_{7}\right\}$ be the Cayley structure of the Cayley projective (or Cayley hyperbolic) plane $\mathbf{O} N^{2}(c)$. Let $(M, f)$ be a submanifold in $\mathbf{F} N^{m}(c)$, where $\mathbf{F}=\mathbf{C}, \mathbf{Q}$ or $\mathbf{O}$ and $m=2$ when $\mathbf{F}=\mathbf{O}$. If $\phi_{i}(T M)=T M(i=1, \ldots, q-1)$, then $(M, f)$ is called an invariant submanifold, where $q=2,4$ or 8 according to whether $\mathbf{F}=\mathbf{C}, \mathbf{Q}$ or $\mathbf{O}$. Note that an invariant submanifold in $\mathbf{Q} N^{m}(c)$ or $\mathbf{O} N^{2}(c)$ is automatically totally geodesic. Let $\mathbf{F}=\mathbf{C}$. If $\phi_{1}\left(T^{\perp} M\right) \subset T M$, then $(M, f)$ is called a generic submanifold. Let $\mathbf{F}=\mathbf{Q}$ or $\mathbf{O}$. If $\phi_{i_{0}}(T M) \subset T M$ for some $i_{0} \in\{1, \ldots, q-1\}$ and $\phi_{i}(T M) \subset T^{\perp} M$ for other $i$, then $(M, f)$ is called a totally complex submanifold. We have the following facts.

\section{PROPOSITION 2.2.}

(i) All submanifolds in a real space form have Lie triple systematic and root decomposable normal bundles.

(ii) All invariant submanifolds in $\mathbf{F} N^{m}(c)(\mathbf{F}=\mathbf{C}, \mathbf{Q}$ or $\mathbf{O})$, all geneic submanifolds in $\mathbf{C} N^{m}(c)$ and all complex m-dimensional totally complex submanifolds in $\mathbf{Q} N^{m}(c)$ have Lie triple systematic and root decomposable normal bundles.

Proof. Statement (i) is trivial. We shall prove statement (ii). Let $\mathbf{F} N^{m}(c)=G / K$ and $(M, f)$ be one of the submanifolds in the statement (ii). Since the ambient space is 
F $N^{m}(c)$, we have

$$
\begin{aligned}
{[[X, Y], Z]=} & -\frac{c}{4}\left\{\langle Y, Z\rangle X-\langle X, Z\rangle Y+\sum_{i=1}^{q-1}\left(\left\langle\phi_{i} Y, Z\right\rangle \phi_{i} X\right.\right. \\
& \left.\left.-\left\langle\phi_{i} X, Z\right\rangle \phi_{i} Y+2\left\langle X, \phi_{i} Y\right\rangle \phi_{i} Z\right)\right\} \quad(X, Y, Z \in \mathfrak{p}) .
\end{aligned}
$$

Thus it can be deduced that $(M, f)$ has a Lie triple systematic normal bundle. Take an arbitrary $\xi \in T_{x}^{\perp} M$, where $f(x)=g K$. Let $\mathfrak{p}=\mathfrak{h}+\sum_{\mu \in \Delta_{+}} \mathfrak{p}_{\mu}$ be the root space decomposition with respect to a maximal abelian subspace $\mathfrak{h}$ in $\mathfrak{p}$ containing $g_{*}^{-1} \xi$. Since the ambient space is $\mathbf{F} N^{m}(c)$, we have $\Delta_{+}=\left\{\sqrt{c}\|\cdot\|, \frac{1}{2} \sqrt{c}\|\cdot\|\right\}$, $\mathfrak{h}=\operatorname{Span}\left\{g_{*}^{-1} \xi\right\}$ and $\mathfrak{p}_{\sqrt{c}\|\cdot\|}=\operatorname{Span}\left\{g_{*}^{-1} \phi_{1} \xi, \ldots, g_{*}^{-1} \phi_{q-1} \xi\right\}$, where $\|\cdot\|$ is the norm associated with the metric of $\mathfrak{p}=T_{e K} N$. It is clear that $g_{*}^{-1}\left(T_{x}^{\perp} M\right)=$ $\mathfrak{h}+\mathfrak{p}_{\sqrt{c}\|\cdot\|} \cap g_{*}^{-1}\left(T_{x}^{\perp} M\right)+\mathfrak{p}_{\frac{1}{2} \sqrt{c}\|\cdot\|} \cap g_{*}^{-1}\left(T_{x}^{\perp} M\right)$. That is, $(M, f)$ has a root decomposable normal bundle.

At the end of this section, we shall explain the curves $Z_{v}$ and $Z_{X, \xi}$ in $\mathfrak{p}$ stated in Theorem A. The curve $Z_{v}$ is the solution of the differential equation

$$
\begin{aligned}
Z^{\prime \prime}(s)= & \operatorname{ad}\left(g_{*}^{-1} \xi\right)^{2}(Z)-2\left[\left[g_{*}^{-1} \xi, g_{*}^{-1} B_{s \xi} B_{\xi}^{-2} \operatorname{grad} \varepsilon\right], g_{*}^{-1} D_{s \xi}^{c o} v_{0}\right] \\
& -2\left[\left[g_{*}^{-1} \xi, s g_{*}^{-1} D_{s \xi}^{s i} v_{0}\right], g_{*}^{-1} \frac{d B_{s \xi}}{d s} B_{\xi}^{-2} \operatorname{grad} \varepsilon\right]
\end{aligned}
$$

satisfying initial conditions

$$
Z(0)=g_{*}^{-1}\left(B_{*}^{-2} v\right) \operatorname{grad} \varepsilon, \quad Z^{\prime}(0)=-g_{*}^{-1}\left(A_{v_{0}} B_{\xi}^{-2}+A_{\xi}\left(B^{-2} v\right)\right) \operatorname{grad} \varepsilon,
$$

where $v_{0}$ is the element of $T_{x}^{\perp} M$ corresponding to $v$ under the natural identification of $T_{\xi}\left(T_{x}^{\perp} M\right)$ and $T_{x}^{\perp} M, B^{-2}{ }_{*}$ is the differential of $B^{-2}$ regarded as a map from $T_{x}^{\perp} M$ to $T_{x}^{*} M \otimes T_{x} M$ and $B^{-2}{ }_{*} v$ is regarded as an element of $T_{x}^{*} M \otimes T_{x} M$ under the natural identification of $T_{B_{\xi}^{-2}}\left(T_{x}^{*} M \otimes T_{x} M\right)$ and $T_{x}^{*} M \otimes T_{x} M$. Note that $v_{0}=\left(D_{\xi}^{s i}\right)^{-1} P_{\gamma_{\xi}}^{-1} v$ holds (see proof of Theorem A). Also, the curve $Z_{X, \xi}$ is the solution of the differential equation

$$
\begin{aligned}
Z^{\prime \prime}= & \operatorname{ad}\left(g_{*}^{-1} \xi\right)^{2}(Z)+\frac{2 X \varepsilon}{\varepsilon(x)} \operatorname{ad}\left(g_{*}^{-1} \xi\right)^{2} g_{*}^{-1} B_{s \xi} B_{\xi}^{-2} \operatorname{grad} \varepsilon \\
& -2\left[\left[g_{*}^{-1} \xi, g_{*}^{-1} B_{s \xi} B_{\xi}^{-2} \operatorname{grad} \varepsilon\right], g_{*}^{-1} \frac{d B_{s \xi}}{d s} X\right] \\
& -2\left[\left[g_{*}^{-1} \xi, g_{*}^{-1} B_{s \xi} X\right], g_{*}^{-1} \frac{d B_{s \xi}}{d s} B_{\xi}^{-2} \operatorname{grad} \varepsilon\right]
\end{aligned}
$$


satisfying initial conditions

$$
\begin{aligned}
Z(0)= & g_{*}^{-1}\left(\pi_{\varepsilon}^{*} \nabla\right)_{\tilde{X}_{\xi}}\left(B^{-2} \operatorname{grad} \varepsilon\right)+g_{*}^{-1} h\left(X, B_{\xi}^{-2} \operatorname{grad} \varepsilon\right), \\
Z^{\prime}(0)= & -\frac{X \varepsilon}{\varepsilon(x)} g_{*}^{-1} A_{\xi} B_{\xi}^{-2} \operatorname{grad} \varepsilon-g_{*}^{-1}\left(\nabla_{X} A\right)_{\xi} B_{\xi}^{-2} \operatorname{grad} \varepsilon \\
& -g_{*}^{-1} A_{\xi}\left(\left(\pi_{\varepsilon}^{*} \nabla\right)_{\tilde{X}_{\xi}}\left(B^{-2} \operatorname{grad} \varepsilon\right)\right)-g_{*}^{-1} h\left(X, A_{\xi} B_{\xi}^{-2} \operatorname{grad} \varepsilon\right) \\
& -\left[\left[g_{*}^{-1} \xi, g_{*}^{-1} X\right], g_{*}^{-1} B_{\xi}^{-2} \operatorname{grad} \varepsilon\right],
\end{aligned}
$$

where $h$ is the second fundamental form of $(M, f)$. Note that $Z_{v}$ and $Z_{X, \xi}$ vanish when $\varepsilon$ is constant.

\section{Proof of Theorem A}

In this section, we shall prove Theorem A. Let $(M, f)$ be a submanifold in a symmetric space $N=G / K$. Denote by $A$ (respectively $A^{\varepsilon}$ ) the shape tensor of $(M, f)$ (respectively $\left(t_{\varepsilon}(M), f_{\varepsilon}\right)$ ). Also, denote by the same symbol $\langle$,$\rangle the metrics of M$, $t_{\varepsilon}(M)$ and $N$. First we shall prepare the following lemma.

LEMMA 3.1. If $(M, f)$ has a root decomposable normal bundle, then the natural lift $\tilde{X}_{\xi}$ of $X \in T_{x} M$ to $\xi \in \pi_{\varepsilon}^{-1}(x)$ can be expressed as

$$
\tilde{X}_{\xi}=P_{\gamma_{\xi}} B_{\xi} X-(X \varepsilon) \bar{E}(\xi),
$$

where $\bar{E}(\xi)=-(1 / \varepsilon(x)) \dot{\gamma}_{\xi}(1)$.

Proof. According to (1.1), we have $\tilde{X}_{\xi}=J_{\xi, X}(1)-(X \varepsilon) \bar{E}(\xi)$. However, according to (1.2), it follows from $J_{\xi, X}(0)=X$ and $J_{\xi, X}^{\prime}(0)=-A_{\xi} X$ that

$$
J_{\xi, X}(1)=P_{\gamma_{\xi}}\left(D_{\xi}^{c o} X-D_{\xi}^{s i} A_{\xi} X\right)
$$

where $f(x)=g K$. The right-hand side can be rewritten as $P_{\gamma_{\xi}} B_{\xi} X$ because $(M, f)$ has root decomposable normal bundle. Hence we can obtain the desired relation.

In the case where $(M, f)$ has a Lie triple systematic and root decomposable normal bundle, we can describe the inward unit normal vector field $E$ of $\left(t_{\varepsilon}(M), f_{\varepsilon}\right)$ as follows.

LEMMA 3.2. If $(M, f)$ has a Lie triple systematic and root decomposable normal bundle, then the inward unit normal vector field $E$ of $\left(t_{\varepsilon}(M), f_{\varepsilon}\right)$ is given by

$$
E(\xi)=\frac{\bar{E}(\xi)+P_{\gamma_{\xi}} B_{\xi}^{-1} \operatorname{grad} \varepsilon}{\sqrt{1+\left\|B_{\xi}^{-1} \operatorname{grad} \varepsilon\right\|^{2}}} \quad\left(\xi \in t_{\varepsilon}(M)\right) .
$$


Proof. For simplicity, set $x:=\pi_{\varepsilon}(\xi)$ and $M_{x}^{\perp}:=\exp ^{\perp}\left(T_{x}^{\perp} M\right)$. Also, denote by $V_{\xi}^{\varepsilon}$ the vertical subspace of $t_{\varepsilon}(M)$ at $\xi$ (i.e. $V_{\xi}^{\varepsilon}=T_{\xi} \pi_{\varepsilon}^{-1}(x)$ ). The tangent space $T_{f_{\varepsilon}(\xi)} M_{x}^{\perp}$ of $M_{x}^{\perp}$ at $f_{\varepsilon}(\xi)$ is decomposed as $T_{f_{\varepsilon}(\xi)} M_{x}^{\perp}=V_{\xi}^{\varepsilon} \oplus \operatorname{Span}\{\bar{E}(\xi)\}$, which is the orthogonal decomposition by Gauss's lemma. Since $(M, f)$ has a Lie triple systematic normal bundle, $M_{x}^{\perp}$ is a totally geodesic submanifold in $N$. Hence the tangent space $T_{f_{\varepsilon}(\xi)} N$ is orthogonally decomposed as $T_{f_{\varepsilon}(\xi)} N=P_{\gamma_{\xi}}\left(T_{x} M\right) \oplus T_{f_{\varepsilon}(\xi)} M_{x}^{\perp}$. Therefore the orthogonal decomposition

$$
T_{f_{\varepsilon}(\xi)} N=P_{\gamma_{\xi}}\left(T_{x} M\right) \oplus V_{\xi}^{\varepsilon} \oplus \operatorname{Span}\{\bar{E}(\xi)\}
$$

is obtained. Now we express $E(\xi)$ as

$$
E(\xi)=\frac{P_{\gamma_{\xi}} Y+Z+\bar{E}(\xi)}{\left\|P_{\gamma_{\xi}} Y+Z+\bar{E}(\xi)\right\|}
$$

where $Y \in T_{x} M$ and $Z \in V_{\xi}^{\varepsilon}$. Since $Z$ is tangent to $f_{\varepsilon}\left(t_{\varepsilon}(M)\right)$ and (3.2) is an orthogonal decomposition, we have $Z=0$. Let $f(x)=g K$. Take an arbitrary $X \in T_{x} M$. From Lemma 3.1, we have

$$
\left\langle E(\xi), \tilde{X}_{\xi}\right\rangle=\frac{\left\langle Y, B_{\xi} X\right\rangle-X \varepsilon}{\left\|P_{\gamma_{\xi}} Y+\bar{E}(\xi)\right\|}=0,
$$

that is, $\left\langle B_{\xi} Y-\operatorname{grad} \varepsilon, X\right\rangle=0$. From the arbitrariness of $X$, we have $B_{\xi} Y=$ $\operatorname{grad} \varepsilon$, that is, $Y=B_{\xi}^{-1} \operatorname{grad} \varepsilon$. Here we note that $B_{\xi}$ is non-singular because $\exp ^{\perp}\left(B_{\varepsilon}(M)\right) \cap F(M, f)=\emptyset$. Therefore, we can obtain the desired relation.

Remark 3.1. In the case where $\varepsilon$ is constant, we can show $E(\xi)=\bar{E}(\xi)$ without the previous assumptions for $(M, f)$.

Further, from these lemmas, we have the following relation.

LemMA 3.3. Assume that $(M, f)$ has a Lie triple systematic and root decomposable normal bundle. Then, for $X \in T_{x} M$ and $\xi \in \pi_{\varepsilon}^{-1}(x)$, the following relation holds:

$$
\left.\operatorname{pr}_{\varepsilon}^{T} P_{\gamma_{\xi}} X=\widetilde{\left(B_{\xi}^{-1} X\right.}\right)_{\xi}-\frac{\left(B_{\xi}^{-1} X\right) \varepsilon}{1+\left\|B_{\xi}^{-1} \operatorname{grad} \varepsilon\right\|^{2}}\left(\widetilde{B_{\xi}^{-2} \operatorname{grad} \varepsilon}\right)_{\xi} .
$$

Denote by $P_{\gamma}, B^{-1} \operatorname{grad} \varepsilon$ the cross section of $f_{\varepsilon}^{*} T N$ defined by assigning $P_{\gamma_{\xi}} B_{\xi}^{-1} \operatorname{grad} \varepsilon$ to each $\xi \in t_{\varepsilon}(M)$. Further, we shall prepare the following lemma.

LEMMA 3.4. (i) For a vertical vector $v$ of $t_{\varepsilon}(M)$ at $\xi \in \pi_{\varepsilon}^{-1}(x)$, we have

$$
\tilde{\nabla}_{v}\left(P_{\gamma} B^{-1} \operatorname{grad} \varepsilon\right)=P_{\gamma_{\xi}} g_{*} Z_{v}(1),
$$

where $f(x)=g K$ and $Z_{v}$ is the curve in $\mathfrak{p}$ explained in Section 2. 
$\left(\hat{\xi}_{0}\right)_{u}$

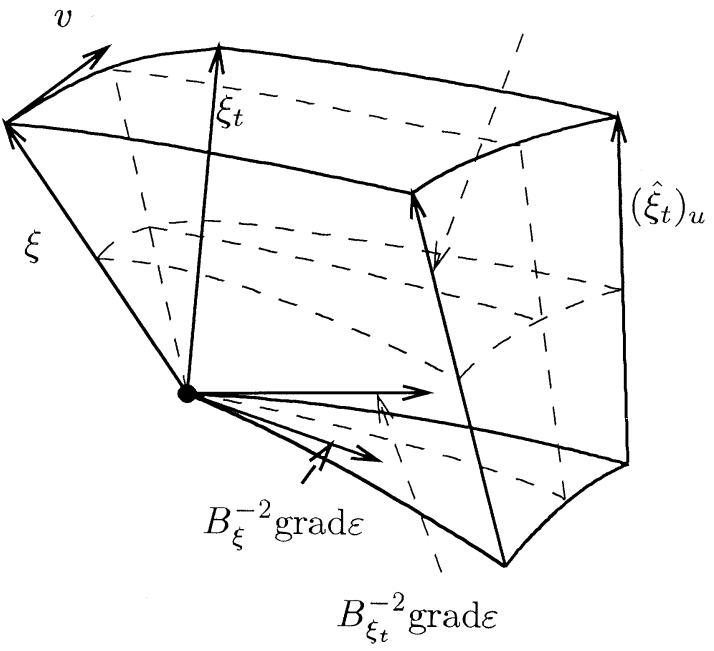

FIGURE 3.

(ii) For $X \in T_{x} M$ and $\xi \in \pi_{\varepsilon}^{-1}(x)$, we have

$$
\tilde{\nabla}_{\tilde{X}_{\xi}}\left(P_{\gamma \cdot} B^{-1} \operatorname{grad} \varepsilon\right)=P_{\gamma_{\xi}} g_{*} Z_{X, \xi}(1),
$$

where $f(x)=g K$ and $Z_{X, \xi}$ is the curve in $\mathfrak{p}$ explained in Section 2.

Proof. First we shall prove statement (i). Let $\xi_{t}\left(0 \leq t \leq t_{0}\right)$ be a curve in $T_{x}^{\perp} M$ with $\dot{\xi}_{0}=v, \beta_{t}(u)\left(0 \leq u \leq u_{t}\right)$ be a curve in $M$ with $\dot{\beta}_{t}(0)=B_{\xi_{t}}^{-2} \operatorname{grad} \varepsilon$ and $\hat{\xi}_{t}$ be the $\nabla^{\perp}$-parallel normal vector field along $\beta_{t}$ with $\left(\hat{\xi}_{t}\right)_{0}=\xi_{t}$. Define a threeparameter map $\delta$ to $N$ by $\delta(t, s, u)=\gamma_{\left(\hat{\xi}_{t}\right)_{u}}(s)$, where $\gamma_{\left(\hat{\xi}_{t}\right)_{u}}(s)$ is the geodesic in $N$ with $\dot{\gamma}_{\left(\hat{\xi}_{t}\right)_{u}}(0)=\left(\hat{\xi}_{t}\right)_{u}$. For simplicity, we denote $\delta_{*}(\partial / \partial t), \delta_{*}(\partial / \partial u)$ and $\delta_{*}(\partial / \partial s)$ by $\partial / \partial t, \partial / \partial u$ and $\partial / \partial s$, respectively and denote $\tilde{\nabla}_{\partial / \partial s}$ by ${ }^{\prime}$.

Set

$$
\tilde{Z}:=\left.\tilde{\nabla}_{\partial / \partial t} \frac{\partial}{\partial u}\right|_{u=t=0} \quad \text { and } \quad Z(s):=g_{*}^{-1} P_{\gamma_{\xi} \mid[0, s]}^{-1} \tilde{Z}(s) .
$$

Denote by $R$ the curvature tensor of $N$. Since $s$-curves are geodesics and $\tilde{\nabla} R=0$, we have

$$
\tilde{Z}^{\prime \prime}=-R\left(\tilde{Z}, \frac{\partial}{\partial s}\right) \frac{\partial}{\partial s}+2 R\left(\frac{\partial}{\partial s}, \frac{\partial}{\partial u}\right) \frac{\partial}{\partial t}^{\prime}+2 R\left(\frac{\partial}{\partial s}, \frac{\partial}{\partial t}\right) \frac{\partial}{\partial u}^{\prime}
$$


in terms of the Jacobi equation and the first Bianchi identity. Hence we have

$$
\begin{aligned}
& Z^{\prime \prime}=\operatorname{ad}\left(g_{*}^{-1} \xi\right)^{2}(Z)-2\left[\left[g_{*}^{-1} \xi, g_{*}^{-1} P_{\left.\gamma_{\xi}\right|_{[0, s]} ^{-1}} \frac{\partial}{\partial u}\right], g_{*}^{-1} P_{\left.\gamma_{\xi}\right|_{[0, s]}}^{-1} \frac{\partial^{\prime}}{\partial t}\right] \\
& -2\left[\left[g_{*}^{-1} \xi, g_{*}^{-1} P_{\left.\gamma_{\xi}\right|_{[0, s]} ^{-1}} \frac{\partial}{\partial t}\right], g_{*}^{-1} P_{\left.\gamma_{\xi}\right|_{[0, s]} ^{-1}} \frac{\partial^{\prime}}{\partial u}\right] \text {. }
\end{aligned}
$$

Since $\left.\frac{\partial}{\partial u}\right|_{u=0}$ is a Jacobi field along $\gamma_{\xi_{t}}$,

$$
\left.\frac{\partial}{\partial u}\right|_{u=s=0}=B_{\xi_{t}}^{-2} \operatorname{grad} \varepsilon \text { and }\left.\frac{\partial^{\prime}}{\partial u}\right|_{u=s=0}=-A_{\xi_{t}} B_{\xi_{t}}^{-2} \operatorname{grad} \varepsilon,
$$

we can obtain

$$
\left.\frac{\partial}{\partial u}\right|_{u=0}=P_{\left.\gamma_{\xi t}\right|_{[0, s]}} B_{s \xi_{t}} B_{\xi_{t}}^{-2} \operatorname{grad} \varepsilon
$$

in terms of (1.2). Hence we also have

$$
\left.\frac{\partial^{\prime}}{\partial u}\right|_{u=t=0}=P_{\left.\gamma_{\xi}\right|_{[0, s]}} \frac{d B_{s \xi}}{d s} B_{\xi}^{-2} \operatorname{grad} \varepsilon
$$

Let $v_{0}$ be the element of $T_{x}^{\perp} M$ corresponding to $v\left(\in T_{\xi}\left(T_{x}^{\perp} M\right)\right)$. Since $\left.\frac{\partial}{\partial t}\right|_{u=t=0}$ is a Jacobi field along $\gamma_{\xi}$,

$$
\left.\frac{\partial}{\partial t}\right|_{u=t=s=0}=0 \quad \text { and }\left.\quad \frac{\partial^{\prime}}{\partial t}\right|_{u=t=s=0}=v_{0}
$$

we can obtain

$$
\left.\frac{\partial}{\partial t}\right|_{u=t=0}=P_{\left.\gamma_{\xi}\right|_{[0, s]}}\left(s \cdot D_{s \xi}^{s i} v_{0}\right)
$$

in terms of (1.2). Hence we also have

$$
\left.\frac{\partial^{\prime}}{\partial t}\right|_{u=t=0}=P_{\left.\gamma_{\xi}\right|_{[0, s]}} D_{s \xi}^{c o} v_{0}
$$

Substituting these relations into (3.3), we see that $Z$ satisfies differential equation (2.2). Also, we have

$$
Z(0)=g_{*}^{-1}\left(\left.\frac{d B_{\xi_{t}}^{-2}}{d t}\right|_{t=0}(\operatorname{grad} \varepsilon)\right)=g_{*}^{-1}\left(B_{*}^{-2} v\right) \operatorname{grad} \varepsilon
$$




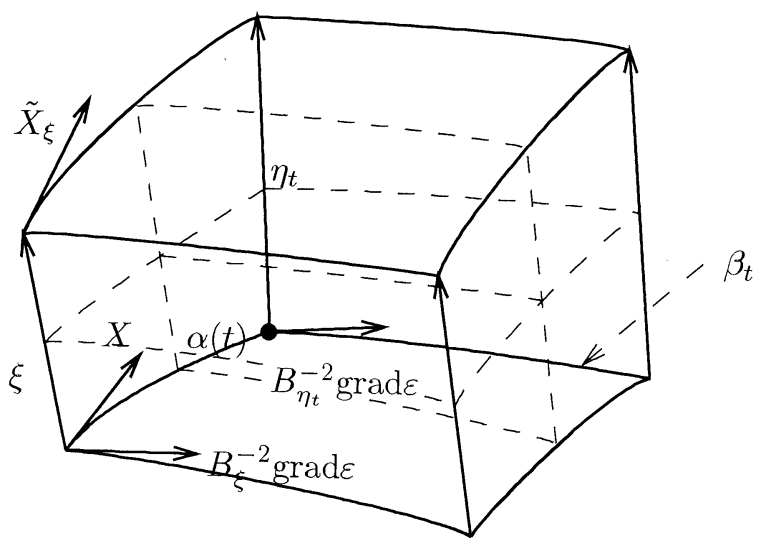

FIGURE 4.

and

$$
\begin{aligned}
Z^{\prime}(0) & =\left.g_{*}^{-1} \tilde{\nabla}_{\partial / \partial t} \tilde{\nabla}_{\partial / \partial s} \frac{\partial}{\partial u}\right|_{u=t=s=0}+\left.g_{*}^{-1} R\left(\frac{\partial}{\partial s}, \frac{\partial}{\partial t}\right) \frac{\partial}{\partial u}\right|_{u=t=s=0} \\
& =\left.g_{*}^{-1} \tilde{\nabla}_{\partial / \partial t} \tilde{\nabla}_{\partial / \partial u} \frac{\partial}{\partial s}\right|_{u=t=s=0}+g_{*}^{-1} R(\xi, 0) B_{\xi}^{-2} \operatorname{grad} \varepsilon \\
& =-g_{*}^{-1} \tilde{\nabla}_{\partial / \partial t}\left(A_{\xi_{t}} B_{\xi_{t}}^{-2} \operatorname{grad} \varepsilon\right)=-g_{*}^{-1}\left(A_{v_{0}} B_{\xi}^{-2}+A_{\xi}\left(B_{*}^{-2} v\right)\right) \operatorname{grad} \varepsilon
\end{aligned}
$$

Thus we have $Z=Z_{v}$. However, it follows from (3.4) that

$$
P_{\gamma_{\xi}} g_{*} Z(1)=\tilde{Z}(1)=\left.\tilde{\nabla}_{\partial / \partial t} \frac{\partial}{\partial u}\right|_{u=t=0, s=1}=\tilde{\nabla}_{v}\left(P_{\gamma \cdot} B^{-1} \operatorname{grad} \varepsilon\right) .
$$

Therefore, we can obtain the desired relation.

Next we shall prove statement (ii). Let $\alpha(t)\left(0 \leq t \leq t_{0}\right)$ be a curve in $M$ with $\dot{\alpha}(0)=X$ and $\hat{\xi}$ be the $\nabla^{\perp}$-parallel normal vector field along $\alpha$ with $\hat{\xi}_{0}=\xi$. Set $\eta_{t}:=(\varepsilon(\alpha(t)) / \varepsilon(x)) \hat{\xi}_{t}$. Let $\beta_{t}(u)\left(0 \leq u \leq u_{t}\right)$ be a curve in $M$ with $\dot{\beta}_{t}(0)=B_{\eta_{t}}^{-2} \operatorname{grad} \varepsilon$ and $\hat{\eta}_{t}$ be the $\nabla^{\perp}$-parallel normal vector field along $\beta_{t}$ with $\left(\hat{\eta}_{t}\right)_{0}=\eta_{t}$. Define a three-parameter map $\delta$ to $N$ by $\delta(t, s, u):=\gamma_{\left(\hat{\eta}_{t}\right)_{u}}(s)$, where $\gamma_{\left(\hat{\eta}_{t}\right)_{u}}$ is the geodesic in $N$ with $\dot{\gamma}_{\left(\hat{\eta}_{t}\right)_{u}}(0)=\left(\hat{\eta}_{t}\right)_{u}$. Set

$$
\tilde{Z}:=\left.\tilde{\nabla}_{\partial / \partial t} \frac{\partial}{\partial u}\right|_{u=t=0} \quad \text { and } \quad Z(s)=g_{*}^{-1} P_{\left.\gamma_{\xi}\right|_{[0, s]} ^{-1}} \tilde{Z}(s) .
$$


Since $s$-curves are geodesics and $\tilde{\nabla} R=0, Z$ satisfies the differential equation (3.3). Since $\left.\frac{\partial}{\partial u}\right|_{u=0}$ is a Jacobi field along $\gamma_{\eta_{t}}$,

$$
\left.\frac{\partial}{\partial u}\right|_{u=s=0}=B_{\eta_{t}}^{-2} \operatorname{grad} \varepsilon \text { and }\left.\frac{\partial^{\prime}}{\partial u}\right|_{u=s=0}=-A_{\eta_{t}} B_{\eta_{t}}^{-2} \operatorname{grad} \varepsilon,
$$

we can obtain

$$
\left.\frac{\partial}{\partial u}\right|_{u=0}=P_{\left.\gamma_{\eta_{t}}\right|_{[0, s]}} B_{s \eta_{t}} B_{\eta_{t}}^{-2} \operatorname{grad} \varepsilon
$$

Hence we also have

$$
\left.\frac{\partial^{\prime}}{\partial u}\right|_{u=0}=P_{\left.\gamma_{\eta_{t}}\right|_{[0, s]}} \frac{d B_{s \eta_{t}}}{d s} B_{\eta_{t}}^{-2} \operatorname{grad} \varepsilon .
$$

Since $\left.\frac{\partial}{\partial t}\right|_{u=t=0}$ is a Jacobi field along $\gamma_{\xi}$,

we can obtain

$$
\left.\frac{\partial}{\partial t}\right|_{u=t=s=0}=X \text { and }\left.\frac{\partial^{\prime}}{\partial t}\right|_{u=t=s=0}=-A_{\xi} X+\frac{X \varepsilon}{\varepsilon(x)} \xi,
$$

and hence

$$
\left.\frac{\partial}{\partial t}\right|_{u=t=0}=P_{\left.\gamma_{\xi}\right|_{[0, s]}} B_{s \xi} X+s \frac{X \varepsilon}{\varepsilon(x)} P_{\left.\gamma_{\xi}\right|_{[0, s]}} \xi
$$

$$
\left.\frac{\partial^{\prime}}{\partial t}\right|_{u=t=0}=P_{\left.\gamma_{\xi}\right|_{[0, s]}} \frac{d B_{s \xi}}{d s} X+\frac{X \varepsilon}{\varepsilon(x)} P_{\left.\gamma_{\xi}\right|_{[0, s]}} \xi .
$$

Substituting these relations into (3.3), we see that $Z$ satisfies differential equation (2.4). We also have

$$
\begin{aligned}
Z(0) & =g_{*}^{-1} \tilde{\nabla}_{X}\left(B_{\eta_{t}}^{-2} \operatorname{grad} \varepsilon\right) \\
& =g_{*}^{-1}\left(\pi_{\varepsilon}^{*} \nabla\right)_{\tilde{X}_{\xi}}\left(B^{-2} \operatorname{grad} \varepsilon\right)+g_{*}^{-1} h\left(X, B_{\xi}^{-2} \operatorname{grad} \varepsilon\right)
\end{aligned}
$$

and

$$
\begin{aligned}
Z^{\prime}(0)= & \left.g_{*}^{-1} \tilde{\nabla}_{\partial / \partial t} \tilde{\nabla}_{\partial / \partial s} \frac{\partial}{\partial u}\right|_{u=t=s=0}+\left.g_{*}^{-1} R\left(\frac{\partial}{\partial s}, \frac{\partial}{\partial t}\right) \frac{\partial}{\partial u}\right|_{u=t=s=0} \\
= & \left.g_{*}^{-1} \tilde{\nabla}_{\partial / \partial t} \tilde{\nabla}_{\partial / \partial u} \frac{\partial}{\partial s}\right|_{u=t=s=0}+g_{*}^{-1} R(\xi, X) B_{\xi}^{-2} \operatorname{grad} \varepsilon \\
= & -g_{*}^{-1} \tilde{\nabla}_{X}\left(A_{\eta_{t}} B_{\eta_{t}}^{-2} \operatorname{grad} \varepsilon\right)-\left[\left[g_{*}^{-1} \xi, g_{*}^{-1} X\right], g_{*}^{-1} B_{\xi}^{-2} \operatorname{grad} \varepsilon\right] \\
= & -\frac{X \varepsilon}{\varepsilon(x)} g_{*}^{-1} A_{\xi} B_{\xi}^{-2} \operatorname{grad} \varepsilon-g_{*}^{-1}\left(\nabla_{X} A\right)_{\xi} B_{\xi}^{-2} \operatorname{grad} \varepsilon \\
& -g_{*}^{-1} A_{\xi}\left(\pi_{\varepsilon}^{*} \nabla\right)_{\tilde{X}_{\xi}}\left(B^{-2} \operatorname{grad} \varepsilon\right) \\
& -g_{*}^{-1} h\left(X, A_{\xi} B_{\xi}^{-2} \operatorname{grad} \varepsilon\right)-\left[\left[g_{*}^{-1} \xi, g_{*}^{-1} X\right], g_{*}^{-1} B_{\xi}^{-2} \operatorname{grad} \varepsilon\right]
\end{aligned}
$$


Thus we have $Z=Z_{X, \xi}$. However, it follows from (3.5) that $P_{\gamma_{\xi}} g_{*} Z(1)=$ $\tilde{\nabla}_{\tilde{X}_{\xi}}\left(P_{\gamma \cdot} B^{-1} \operatorname{grad} \varepsilon\right)$. Therefore, we can obtain the desired relation.

Now we shall prove Theorem A in terms of these lemmas.

Proof of Theorem A. First we shall prove statement (i). Set $\bar{v}:=\varepsilon(x) v /\|v\|$. Define a geodesic variation $\delta$ by $\delta(t, s)=\exp ^{\perp}\left(s \cdot\left(\cos t \cdot \xi+\sin t \cdot \bar{v}_{0}\right)\right)$, where $\bar{v}_{0}$ is the element of $T_{x}^{\perp} M$ corresponding to $\bar{v}\left(\in T_{\xi}\left(T_{x}^{\perp} M\right)\right)$. For simplicity, we denote $\delta_{*}(\partial / \partial t)$ (respectively $\delta_{*}(\partial / \partial s)$ ) by $\partial / \partial t$ (respectively $\partial / \partial s$ ). Also, we set $J:=\left.\frac{\partial}{\partial t}\right|_{t=0}$. It is clear that $\bar{v}=J(1)$ and

$$
\tilde{\nabla}_{\bar{v}} \bar{E}=-\left.\frac{1}{\varepsilon(x)} \tilde{\nabla}_{\partial / \partial t} \frac{\partial}{\partial s}\right|_{t=0, s=1}=-\left.\frac{1}{\varepsilon(x)} \tilde{\nabla}_{\partial / \partial s} \frac{\partial}{\partial t}\right|_{t=0, s=1}=-\frac{1}{\varepsilon(x)} J^{\prime}(1),
$$

where $J^{\prime}=\tilde{\nabla}_{\partial / \partial s} J$. Since $J$ is a Jacobi field along $\gamma_{\xi}$,

$$
J(0)=0 \quad \text { and } \quad J^{\prime}(0)=\left.\tilde{\nabla}_{\partial / \partial t} \frac{\partial}{\partial s}\right|_{t=s=0}=\bar{v}_{0},
$$

we have $J(s)=s \cdot P_{\left.\gamma_{\xi}\right|_{[0, s]}} D_{s \xi}^{s i} \bar{v}_{0}$ in terms of (1.2). Hence we have $J^{\prime}(1)=P_{\gamma_{\xi}} D_{\xi}^{c o} \bar{v}_{0}$. Thus we can obtain

$$
\tilde{\nabla}_{\bar{v}} \bar{E}=-\frac{1}{\varepsilon(x)} P_{\gamma_{\xi}} D_{\xi}^{c o} \bar{v}_{0} .
$$

Also, we have $\bar{v}=J(1)=P_{\gamma_{\xi}} D_{\xi}^{s i} \bar{v}_{0}$, that is, $\bar{v}_{0}=\left(D_{\xi}^{s i}\right)^{-1} P_{\gamma_{\xi}}^{-1} \bar{v}$. Therefore, we can obtain

$$
\tilde{\nabla}_{v} \bar{E}=-\frac{1}{\varepsilon(x)} P_{\gamma_{\xi}} D_{\xi}^{c o}\left(D_{\xi}^{s i}\right)^{-1} P_{\gamma_{\xi}}^{-1} v
$$

which is equal to $-\left(\mu\left(g_{*}^{-1} \xi\right) / \varepsilon(x) \tan \mu\left(g_{*}^{-1} \xi\right)\right) v$ because $g_{*}^{-1} P_{\gamma_{\xi}}^{-1} v$ is a root vector for a root $\mu$. Therefore, from Lemma 3.2, we can obtain

$$
\begin{aligned}
\tilde{\nabla}_{v} E \equiv & \frac{1}{\sqrt{1+\left\|B_{\xi}^{-1}\left(\operatorname{grad} \varepsilon_{x}\right)\right\|^{2}}}\left\{-\frac{\mu\left(g_{*}^{-1} \xi\right)}{\varepsilon(x) \tan \mu\left(g_{*}^{-1} \xi\right)} v+\operatorname{pr}_{\varepsilon}^{T}\left(\tilde{\nabla}_{v}\left(P_{\gamma \cdot} B^{-1} \operatorname{grad} \varepsilon\right)\right)\right\} \\
& (\bmod \operatorname{Span}\{E(\xi)\}),
\end{aligned}
$$

which together with (i) of Lemma 3.4 gives the relation $(0.1)$.

Next we shall prove statement (ii). Let $X \in T_{x} M$ as in statement (ii). Take a curve $\beta$ in $M$ with $\dot{\beta}(0)=X$. Let $\tilde{\xi}$ be a $\nabla^{\perp}$ - parallel vector field along $\beta$ with $\tilde{\xi}(0)=\xi$ and $\tilde{\beta}$ be a curve in $t_{\varepsilon}(M)$ defined by $\tilde{\beta}(t)=((\varepsilon(\beta(t)) / \varepsilon(x)) \tilde{\xi}(t)$. Define a rectangle $\delta$ by $\delta(t, s):=\gamma_{\tilde{\beta}(t)}(s)$, where $\gamma_{\tilde{\beta}(t)}$ is the geodesic in $N$ with $\dot{\gamma}_{\tilde{\beta}(t)}(0)=\tilde{\beta}(t)$. 
For simplicity, we denote $\delta_{*}(\partial / \partial t)$ (respectively $\delta_{*}(\partial / \partial s)$ ) by $\partial / \partial t$ (respectively $\partial / \partial s)$. Clearly we have

$$
\left.\frac{\partial}{\partial t}\right|_{(0,1)}=\dot{\tilde{\beta}}(0)=\tilde{X}_{\xi} \quad \text { and }\left.\quad \frac{\partial}{\partial s}\right|_{(t, 1)}=-\varepsilon(\beta(t)) \bar{E}(\tilde{\beta}(t))
$$

Hence we have

$$
\tilde{\nabla}_{\tilde{X}_{\xi}} \bar{E}=-\frac{X \varepsilon}{\varepsilon(x)} \bar{E}(\xi)-\left.\frac{1}{\varepsilon(x)}\left(\tilde{\nabla}_{\frac{\partial}{\partial s}} \frac{\partial}{\partial t}\right)\right|_{(0,1)}
$$

Since $\delta$ is a geodesic variation, $J:=\left.\frac{\partial}{\partial t}\right|_{(0, \cdot)}$ is a Jacobi field. Also, we have $J(0)=X$ and

$$
J^{\prime}(0)=\left.\left(\tilde{\nabla}_{\partial / \partial s} \frac{\partial}{\partial t}\right)\right|_{(0,0)}=\left.\left(\tilde{\nabla}_{\partial / \partial t} \frac{\partial}{\partial s}\right)\right|_{(0,0)}=\tilde{\nabla}_{X}\left(\frac{\varepsilon(\beta(t))}{\varepsilon(x)} \tilde{\xi}_{t}\right)=\frac{X \varepsilon}{\varepsilon(x)} \xi-A_{\xi} X .
$$

Hence it follows from (1.2) that

$$
J(s)=P_{\left.\gamma_{\xi}\right|_{[0, s]}}\left(B_{s \xi} X+s \frac{X \varepsilon}{\varepsilon(x)} \xi\right) .
$$

Differentiating this relation, we have

$$
\left.\left(\tilde{\nabla}_{\partial / \partial s} \frac{\partial}{\partial t}\right)\right|_{(0,1)}=J^{\prime}(1)=\left.P_{\gamma_{\xi}} \frac{d B_{s \xi}}{d s}\right|_{s=1} X-(X \varepsilon) \bar{E}(\xi) .
$$

Substituting this relation into (3.6), we have

$$
\tilde{\nabla}_{\tilde{X}_{\xi}} \bar{E}=-\left.\frac{1}{\varepsilon(x)} P_{\gamma_{\xi}} \frac{d B_{s \xi}}{d s}\right|_{s=1} X,
$$

which is equal to

$$
\frac{1}{\varepsilon(x)}\left(\lambda \cos \mu\left(g_{*}^{-1} \xi\right)+\mu\left(g_{*}^{-1} \xi\right) \sin \mu\left(g_{*}^{-1} \xi\right)\right) P_{\gamma_{\xi}} X
$$

because $A_{\xi} X=\lambda X$ and $g_{*}^{-1} X \in \mathfrak{p}_{\mu}$. It also follows from Lemma 3.1 that

$$
P_{\gamma_{\xi}} X=\frac{\mu\left(g_{*}^{-1} \xi\right)}{\mu\left(g_{*}^{-1} \xi\right) \cos \mu\left(g_{*}^{-1} \xi\right)-\lambda \sin \mu\left(g_{*}^{-1} \xi\right)}\left(\tilde{X}_{\xi}+(X \varepsilon) \bar{E}(\xi)\right) .
$$

Therefore, we can obtain

$$
\tilde{\nabla}_{\tilde{X}_{\xi}} \bar{E}=\frac{\lambda+\mu\left(g_{*}^{-1} \xi\right) \tan \mu\left(g_{*}^{-1} \xi\right)}{\varepsilon(x)\left(1-\lambda \tan \mu\left(g_{*}^{-1} \xi\right) / \mu\left(g_{*}^{-1} \xi\right)\right)}\left(\tilde{X}_{\xi}+(X \varepsilon) \bar{E}(\xi)\right) .
$$


However, from Lemma 3.2 it can be deduced that

$\tilde{\nabla}_{\tilde{X}_{\xi}} E \equiv \frac{1}{\sqrt{1+\left\|B_{\xi}^{-1} \operatorname{grad} \varepsilon\right\|^{2}}}\left(\tilde{\nabla}_{\tilde{X}_{\xi}} \bar{E}+\tilde{\nabla}_{\tilde{X}_{\xi}}\left(P_{\gamma,} B^{-1} \operatorname{grad} \varepsilon\right)\right) \quad(\bmod \operatorname{Span}\{E(\xi)\})$.

This together with (3.7) and (ii) of Lemma 3.4 gives relation (0.2), where we note that

$$
\operatorname{pr}_{\varepsilon}^{T} \bar{E}(\xi)=-\frac{1}{1+\left\|B_{\xi}^{-1} \operatorname{grad} \varepsilon\right\|^{2}}\left(B_{\xi}^{-2} \operatorname{grad} \varepsilon\right)_{\xi}
$$

because of Lemmas 3.2 and 3.3 .

\section{Proof of Theorem B}

In this section, we shall prove Theorem B. Let $(M, f)$ be a totally geodesic submanifold in the $m$-dimensional real space form $N^{m}(c)$ of constant curvature $c$. Let $N^{m}(c)=G / K$. First we shall prepare the following lemma.

LEMMA 4.1.

(i) For a vertical vector $v$ of $t_{\varepsilon}(M)$, we have $Z_{v}=0$.

(ii) For $X \in T_{x} M$ and $\xi \in \pi_{\varepsilon}^{-1}(x)$, we have

$$
\begin{aligned}
g_{*} Z_{X, \xi}(1)= & \frac{(X \varepsilon) \sqrt{c} \sin (\sqrt{c} \varepsilon(x))}{\cos ^{2}(\sqrt{c} \varepsilon(x))} \operatorname{grad} \varepsilon+\frac{1}{\cos (\sqrt{c} \varepsilon(x))} \nabla_{X} \operatorname{grad} \varepsilon \\
& +\frac{X \varepsilon}{2 \varepsilon(x) \cos ^{2}(\sqrt{c} \varepsilon(x))}(4 c \varepsilon(x)+\sqrt{c} \sin (2 \sqrt{c} \varepsilon(x))) \xi,
\end{aligned}
$$

where $f(x)=g K$.

Proof. Since the ambient space is of constant curvature $c$ and $(M, f)$ is totally geodesic, we have

$$
\begin{aligned}
D_{s \xi}^{c o} & =\mathrm{pr}_{0}+\cos (\sqrt{c} \varepsilon(x) s) \mathrm{pr}_{1}, \\
D_{s \xi}^{s i} & =\mathrm{pr}_{0}+\frac{\sin (\sqrt{c} \varepsilon(x) s)}{\sqrt{c} \varepsilon(x) s} \mathrm{pr}_{1}, \\
B_{s \xi} & =\cos (\sqrt{c} \varepsilon(x) s) I, \quad B^{-2}{ }_{*} v=0
\end{aligned}
$$

for $\xi \in T_{x}^{\perp} M$ and $v \in V_{\xi}^{\varepsilon}$, where $f(x)=g K, \operatorname{pr}_{0}$ (respectively $\mathrm{pr}_{1}$ ) is the projection of $T_{f(x)} N^{m}(c)$ onto $\operatorname{Span}\{\xi\}$ (respectively $\operatorname{Span}\{\xi\}^{\perp}$ ) and $I$ is the identity transformation of $T_{x} M$. Also, we have [[ $\left.\left.Y_{1}, Y_{2}\right], Y_{3}\right]=c\left(\left\langle Y_{1}, Y_{3}\right\rangle Y_{2}-\left\langle Y_{2}, Y_{3}\right\rangle Y_{1}\right)$ for $Y_{i} \in \mathfrak{p}(i=1,2,3)$. By solving the differential equation (2.2) (respectively (2.4)) 
under the initial condition (2.3) (respectively (2.5)) in terms of these relations, we can obtain the relation in (i) (respectively (ii)).

Remark 4.1. For a general submanifold in $N^{m}(c)$, we can concretely describe $Z_{v}$ and $Z_{X, \xi}$.

Now we shall prove Theorem B in terms of Theorem A, this lemma, Lemmas 3.2 and 3.3.

Proof of Theorem B. Fix $\xi \in \pi_{\varepsilon}^{-1}\left(\gamma\left(s_{0}\right)\right)$. Let $\gamma\left(s_{0}\right)=g K$. The maximal abelian subspace $\mathfrak{h}$ containing $g_{*}^{-1} \xi$ is given by $\mathfrak{h}:=\operatorname{Span}\left\{g_{*}^{-1} \xi\right\}$ and the positive root system consists of only $\sqrt{c}\|\cdot\|$, where $\|\cdot\|$ is the norm of $\mathfrak{h}$. Let $v$ be a vertical vector of $t_{\varepsilon}(M)$ at $\xi$. Since $g_{*}^{-1} P_{\gamma_{\xi}}^{-1} v$ is a root vector for $\sqrt{c}\|\cdot\|$, it can be deduced from Theorem A, Lemma 4.1 and (4.1) that

$$
A_{E(\xi)}^{\varepsilon} v=\frac{\cos \left(\sqrt{c} \varepsilon\left(s_{0}\right)\right)}{\sqrt{\cos ^{2}\left(\sqrt{c} \varepsilon\left(s_{0}\right)\right)+\varepsilon^{\prime}\left(s_{0}\right)^{2}}} \frac{\sqrt{c}}{\tan \left(\sqrt{c} \varepsilon\left(s_{0}\right)\right)} v .
$$

Similarly, since $g_{*}^{-1} \dot{\gamma}\left(s_{0}\right)$ is a root vector for $\sqrt{c}\|\cdot\|$, we have

$$
\begin{aligned}
A_{E(\xi)}^{\varepsilon} \widetilde{\dot{\gamma}\left(s_{0}\right)_{\xi}}= & \frac{-\cos \left(\sqrt{c} \varepsilon\left(s_{0}\right)\right)}{\left(\cos ^{2}\left(\sqrt{c} \varepsilon\left(s_{0}\right)\right)+\varepsilon^{\prime}\left(s_{0}\right)^{2}\right)^{3 / 2}} \\
& \times\left\{\varepsilon^{\prime \prime}\left(s_{0}\right)+\sqrt{c} \tan \left(\sqrt{c} \varepsilon\left(s_{0}\right)\right) \varepsilon^{\prime}\left(s_{0}\right)^{2}+\frac{2 c \varepsilon\left(s_{0}\right)}{\cos ^{2}\left(\sqrt{c} \varepsilon\left(s_{0}\right)\right)} \varepsilon^{\prime}\left(s_{0}\right)^{2}\right. \\
& \left.+\sqrt{c} \tan \left(\sqrt{c} \varepsilon\left(s_{0}\right)\right) \varepsilon^{\prime}\left(s_{0}\right)+\frac{\sqrt{c}}{2} \sin \left(2 \sqrt{c} \varepsilon\left(s_{0}\right)\right)\right\} \widetilde{\gamma\left(s_{0}\right)_{\xi}},
\end{aligned}
$$

where we have used Theorem A, Lemmas 3.2, 3.3 and 4.1. Hence the mean curvature $H_{\xi}$ of $\left(t_{\varepsilon}\left(M_{\gamma}\right), \gamma_{\varepsilon}\right)$ at $\xi$ can be described as

$$
\begin{aligned}
H_{\xi}= & \frac{-\cos \left(\sqrt{c} \varepsilon\left(s_{0}\right)\right)}{(m-1)\left(\cos ^{2}\left(\sqrt{c} \varepsilon\left(s_{0}\right)\right)+\varepsilon^{\prime}\left(s_{0}\right)^{2}\right)^{3 / 2}} \\
& \times\left\{\varepsilon^{\prime \prime}\left(s_{0}\right)+\sqrt{c} \tan \left(\sqrt{c} \varepsilon\left(s_{0}\right)\right) \varepsilon^{\prime}\left(s_{0}\right)^{2}+\frac{2 c \varepsilon\left(s_{0}\right)}{\cos ^{2}\left(\sqrt{c} \varepsilon\left(s_{0}\right)\right)} \varepsilon^{\prime}\left(s_{0}\right)^{2}\right. \\
& -\frac{(m-2) \sqrt{c}}{\tan \left(\sqrt{c} \varepsilon\left(s_{0}\right)\right)} \varepsilon^{\prime}\left(s_{0}\right)^{2}+\sqrt{c} \tan \left(\sqrt{c} \varepsilon\left(s_{0}\right)\right) \varepsilon^{\prime}\left(s_{0}\right)+\frac{\sqrt{c}}{2} \sin \left(2 \sqrt{c} \varepsilon\left(s_{0}\right)\right) \\
& \left.-\frac{(m-2) \sqrt{c} \cos ^{2}\left(\sqrt{c} \varepsilon\left(s_{0}\right)\right)}{\tan \left(\sqrt{c} \varepsilon\left(s_{0}\right)\right)}\right\} .
\end{aligned}
$$

Thus we can obtain the statement of Theorem B. 


\section{Proof of Theorem $\mathrm{C}$}

In this section, we shall prove Theorem C. Let $(M, f)$ be a totally geodesic invariant submanifold in $\mathbf{F} N^{m}(c)$, where $\mathbf{F}=\mathbf{C}, \mathbf{Q}$ or $\mathbf{O}$ and $m=2$ when $\mathbf{F}=\mathbf{O}$. Let $\mathbf{F} N^{m}(c)=G / K$. First we shall prepare the following lemma.

LEMMA 5.1.

(i) Let $v$ be a vertical vector of $t_{\varepsilon}(M)$ at $\xi \in \pi_{\varepsilon}^{-1}(x)$. If $P_{\gamma_{\xi}}^{-1} v \in \operatorname{Span}\left\{\phi_{1} \xi, \ldots\right.$, $\left.\phi_{q-1} \xi\right\}$, then we have

$$
g_{*} Z_{v}(1)=\frac{\cos \frac{3}{2} \sqrt{c} \varepsilon(x)-\cos \frac{1}{2} \sqrt{c} \varepsilon(x)}{4 \varepsilon(x)^{2} \cos ^{2} \frac{1}{2} \sqrt{c} \varepsilon(x)} \sum_{i=1}^{q-1}\left\langle\phi_{i} \xi, v_{0}\right\rangle \phi_{i} \operatorname{grad} \varepsilon,
$$

where $f(x)=g K$ and $v_{0}$ is the element of $T_{x}^{\perp} M$ corresponding to $v$ under the natural identification of $T_{\xi}\left(T_{x}^{\perp} M\right)$ and $T_{x}^{\perp} M$. Also, if $P_{\gamma_{\xi}}^{-1} v \in$ $\operatorname{Span}\left\{\phi_{1} \xi, \ldots, \phi_{q-1} \xi\right\}^{\perp}$, then we have $g_{*} Z_{v}(1)=0$.

(ii) For $X \in T_{x} M$ and $\xi \in \pi_{\varepsilon}^{-1}(x)$, we have

$$
\begin{aligned}
g_{*} Z_{X, \xi}(1)= & \frac{1}{\cos \frac{1}{2} \sqrt{c} \varepsilon(x)} \nabla_{X} \operatorname{grad} \varepsilon+\frac{(X \varepsilon) \sqrt{c} \sin (\sqrt{c} \varepsilon(x))}{4 \varepsilon(x) \cos ^{2} \frac{1}{2} \sqrt{c} \varepsilon(x)} \xi \\
& +\sum_{i=1}^{q-1} \frac{\left(\left(\phi_{i} X\right) \varepsilon\right) \sqrt{c} \sin (\sqrt{c} \varepsilon(x))}{4 \varepsilon(x) \cos ^{2} \frac{1}{2} \sqrt{c} \varepsilon(x)} \phi_{i} \xi,
\end{aligned}
$$

where $f(x)=g K$.

Proof. Since $(M, f)$ is a totally geodesic invariant submanifold in $\mathbf{F} N^{m}(c)$, we have

$$
\begin{aligned}
& D_{s \xi}^{c o}=\operatorname{pr}_{0}+\sum_{k=1}^{2} \cos \frac{\sqrt{c} \varepsilon(x) s}{k} \operatorname{pr}_{k}, \\
& D_{s \xi}^{s i}=\operatorname{pr}_{0}+\sum_{k=1}^{2} \frac{k \sin (\sqrt{c} \varepsilon(x) s / k)}{\sqrt{c} \varepsilon(x) s} \operatorname{pr}_{k}, \\
& B_{s \xi}=\cos \frac{\sqrt{c} \varepsilon(x) s}{2} I, \quad B^{-2} v=0,
\end{aligned}
$$

where $\operatorname{pr}_{0}, \operatorname{pr}_{1}$ and $\mathrm{pr}_{2}$ are the projections of $T_{f(x)} \mathbf{C} N^{m}(c)$ onto $\operatorname{Span}\{\xi\}$, $\operatorname{Span}\left\{\phi_{1} \xi, \ldots, \phi_{q-1} \xi\right\}$ and $\operatorname{Span}\left\{\xi, \phi_{1} \xi, \ldots, \phi_{q-1} \xi\right\}^{\perp}$, respectively. By solving the differential equation (2.2) (respectively (2.4)) under the initial condition (2.3) (respectively (2.5)) in terms of these relations and (2.1), we can obtain the relations in (i) (respectively (ii)). 
Remark 5.1. The vector $v_{0}$ in this lemma is described as

$$
v_{0}=\left(D_{\xi}^{s i}\right)^{-1} P_{\gamma_{\xi}}^{-1} v=\left\{\begin{array}{l}
\frac{\sqrt{c} \varepsilon(x)}{\sin (\sqrt{c} \varepsilon(x))} P_{\gamma_{\xi}}^{-1} v, \quad\left(v_{0} \in \operatorname{Span}\left\{\phi_{1} \xi, \ldots, \phi_{q-1} \xi\right\}\right) \\
\frac{\sqrt{c} \varepsilon(x)}{2 \sin \frac{1}{2} \sqrt{c} \varepsilon(x)} P_{\gamma_{\xi}}^{-1} v, \quad\left(v_{0} \in \operatorname{Span}\left\{\phi_{1} \xi, \ldots, \phi_{q-1} \xi\right\}^{\perp}\right) .
\end{array}\right.
$$

Now we shall prove Theorem $\mathrm{C}$ in terms of Theorem A, this lemma, Lemmas 3.2 and 3.3.

Proof of Theorem $C$. Fix $\xi \in \pi_{\varepsilon}^{-1}\left(\gamma\left(z_{0}\right)\right)$. Let $\gamma\left(z_{0}\right)=g K$. The maximal abelian subspace $\mathfrak{h}$ containing $g_{*}^{-1} \xi$ is given by $\mathfrak{h}:=\operatorname{Span}\left\{g_{*}^{-1} \xi\right\}$, the positive root system is consisted of $\sqrt{c}\|\cdot\|$ and $\frac{1}{2} \sqrt{c}\|\cdot\|$, and the root space for $\sqrt{c}\|\cdot\|$ (respectively $\frac{1}{2} \sqrt{c}\|\cdot\|$ ) is given by $\operatorname{Span}\left\{g_{*}^{-1} \phi_{1} \xi, \ldots, g_{*}^{-1} \phi_{q-1} \xi\right\}$ (respectively $\operatorname{Span}\left\{g_{*}^{-1} \phi_{1} \xi, \ldots, g_{*}^{-1} \phi_{q-1} \xi\right\}^{\perp}$ ), where $\|\cdot\|$ is the norm of $\mathfrak{h}$. Let $v$ be a vertical vector of $t_{\varepsilon}(M)$ at $\xi$. If $P_{\gamma_{\xi}}^{-1} v \in \operatorname{Span}\left\{\phi_{1} \xi, \ldots, \phi_{q-1} \xi\right\}$, then $g_{*}^{-1} P_{\gamma_{\xi}}^{-1} v$ is a root vector for $\sqrt{c}\|\cdot\|$. Hence it follows from Theorem A, Lemmas 5.1 and 3.3 and (5.1) that

$$
A_{E(\xi)}^{\varepsilon} v \equiv \frac{\cos \frac{1}{2} \sqrt{c} \varepsilon(x)}{\sqrt{\cos ^{2} \frac{1}{2} \sqrt{c} \varepsilon(x)+\|\operatorname{grad} \varepsilon\|^{2}}} \frac{\sqrt{c}}{\tan (\sqrt{c} \varepsilon(x))} v \quad\left(\bmod H_{\xi}^{\varepsilon}\right)
$$

Also, if $P_{\gamma_{\xi}}^{-1} v \in \operatorname{Span}\left\{\phi_{1} \xi, \ldots, \phi_{q-1} \xi\right\}^{\perp}$, then $g_{*}^{-1} P_{\gamma_{\xi}}^{-1} v$ is a root vector for $\frac{1}{2} \sqrt{c}\|\cdot\|$. Hence it follows from Theorem A, Lemma 5.1 and (5.1) that

$$
A_{E(\xi)}^{\varepsilon} v=\frac{\cos \frac{1}{2} \sqrt{c} \varepsilon(x)}{\sqrt{\cos ^{2} \frac{1}{2} \sqrt{c} \varepsilon(x)+\|\operatorname{grad} \varepsilon\|^{2}}} \cdot \frac{\sqrt{c}}{2 \tan \frac{1}{2} \sqrt{c} \varepsilon(x)} v .
$$

Further, according to Theorem A, Lemmas 5.1, 3.2, 3.3 and (5.1), we have

$$
\begin{aligned}
& A_{E(\xi)}^{\varepsilon}\left(\frac{\widetilde{\partial}}{\partial x_{i}}\right)_{\xi} \equiv \frac{\cos \frac{1}{2} \sqrt{c} \varepsilon(x)}{\sqrt{\cos ^{2} \frac{1}{2} \sqrt{c} \varepsilon}(x)+\|\operatorname{grad} \varepsilon\|^{2}}\left\{-\frac{1}{2} \sqrt{c} \tan \frac{1}{2} \sqrt{c} \varepsilon(x)\left(\frac{\widetilde{\partial}}{\partial x_{i}}\right)_{\xi}\right. \\
& +\frac{\frac{1}{2} \sqrt{c} \varepsilon_{x_{i}} \sin (\sqrt{c} \varepsilon(x))+\sqrt{c} \varepsilon_{x_{i}} \cos \frac{1}{2} \sqrt{c} \varepsilon(x)+\sum_{j=0}^{q-1}\left(1 / \rho^{2}\right)\left(\varepsilon_{x_{i} x_{j}}-\sum_{k=0}^{q-1} \Gamma_{i j}^{k} \varepsilon_{x_{k}}\right) \varepsilon_{x_{j}}}{\cos ^{2} \frac{1}{2} \sqrt{c} \varepsilon(x)\left(\cos ^{2} \frac{1}{2} \sqrt{c} \varepsilon(x)+\|\operatorname{grad} \varepsilon\|^{2}\right)}
\end{aligned}
$$




$$
\begin{aligned}
& \times \sum_{l=0}^{q-1} \frac{\varepsilon_{x_{l}}}{\rho^{2}}\left(\frac{\widetilde{\partial}}{\partial x_{l}}\right)_{\xi}-\frac{1}{\cos ^{2} \frac{1}{2} \sqrt{c} \varepsilon(x)} \sum_{j=0}^{q-1} \frac{1}{\rho^{2}} \\
& \left.\times\left(\varepsilon_{x_{i} x_{j}}-\sum_{k=0}^{q-1} \Gamma_{i j}^{k} \varepsilon_{x_{k}}\right)\left(\frac{\widetilde{\partial}}{\partial x_{j}}\right)_{\xi}\right\} \quad\left(\bmod V_{\xi}^{\varepsilon}\right) .
\end{aligned}
$$

By calculating the mean curvature vector $H_{\xi}\left(=(1 / q m-1) \operatorname{Tr} A_{E(\xi)}^{\varepsilon}\right)$ at $\xi$ in terms of these relations, we can obtain the statement of Theorem $\mathrm{C}$.

\section{Concluding remarks}

In this section, in the case where $(M, f)$ is a principal orbit of the isotropy representation of a symmetric space, we shall explain that the curves $Z_{v}$ and $Z_{X, \xi}$ stated in Section 2 are concretely described. We shall also investigate the property of a constant tube over the principal orbit. Let $N=G / K$ be a symmetric space, $(\mathfrak{g}, \tau)$ be its orthogonal symmetric Lie algebra and $\mathfrak{p}$ (respectively $\mathfrak{f}$ ) be the eigenspace of $\tau$ for -1 (respectively 1). Fix a maximal abelian subspace $\mathfrak{h}$ of $\mathfrak{p}$ and let $\mathfrak{p}=\mathfrak{h}+\sum_{\mu \in \Delta_{+}} \mathfrak{p}_{\mu}$ be the root space decomposition with respect to $\mathfrak{h}$. Let $Y$ be a regular element of $\mathfrak{h}$. Let $M$ be the principal orbit through $\exp (Y)$ of the isotropy representation of $N$ and $\varepsilon$ be a sufficiently small positive function on $M$, where exp is the exponential map of $N$. Define distributions $D_{\mu}\left(\mu \in \triangle_{+}\right)$on $M$ by assigning $g_{*} \mathfrak{p}_{\mu}$ to each $x \in M$, where $g$ is an element of $G$ satisfying $x=g K$ and $g_{*}^{-1} T_{x}^{\perp} M=\mathfrak{h}$. Clearly we have $T M=\oplus_{\mu \in \triangle_{+}} D_{\mu}$ (orthogonal direct sum). Denote by $\operatorname{pr}_{\mu}$ the projection of $T M$ onto $D_{\mu}$. The shape operator $A_{\xi}\left(\xi \in T_{x}^{\perp} M\right)$ of $M$ is described as

$$
A_{\xi}=\sum_{\mu \in \Delta_{+}} \frac{\mu\left(g_{*}^{-1} \xi\right)}{\tan \mu(Y)} \mathrm{pr}_{\mu} \text {. }
$$

Also, we have

$$
\begin{aligned}
& D_{s \xi}^{c o}=\operatorname{pr}_{\perp}+\sum_{\mu \in \Delta_{+}} \cos \left(s \mu\left(g_{*}^{-1} \xi\right)\right) \tilde{\mathrm{pr}}_{\mu}, \\
& D_{s \xi}^{s i}=\operatorname{pr}_{\perp}+\sum_{\mu \in \Delta_{+}} \frac{\sin \left(s \mu\left(g_{*}^{-1} \xi\right)\right)}{s \mu\left(g_{*}^{-1} \xi\right)} \tilde{\mathrm{pr}}_{\mu} .
\end{aligned}
$$

Here $\mathrm{pr}_{\perp}$ is the projection of $\iota^{*} T N$ onto $T^{\perp} M$ and $\tilde{\mathrm{pr}}_{\mu}$ is the projection of $\iota^{*} T N$ onto $D_{\mu}$, where $\iota$ is the inclusion map of $M$ into $N$. Hence we have

$$
B_{s \xi}=\sum_{\mu \in \Delta_{+}}\left(\cos \left(s \mu\left(g_{*}^{-1} \xi\right)\right)-\frac{\sin \left(s \mu\left(g_{*}^{-1} \xi\right)\right)}{\tan \mu(Y)}\right) \operatorname{pr}_{\mu} .
$$


In terms of these relations, we can solve differential equations (2.2) and (2.4). That is, we can concretely describe the curves $Z_{v}$ and $Z_{X, \xi}$ stated in Section 2 . Hence the shape operator of the $\varepsilon$-tube over the principal orbit is concretely described. It is expected that the explicit description will be useful in finding the condition (related to $\varepsilon$ ) for the tube over the principal orbit to satisfy various geometric properties.

In particular, we shall investigate the property of a constant tube over the principal orbit. Assume that $\varepsilon$ is constant. Since

$$
A_{\xi}=\sum_{\mu \in \Delta_{+}} \frac{\mu\left(g_{*}^{-1} \xi\right)}{\tan \mu(Y)} \operatorname{pr}_{\mu} \quad\left(\xi \in t_{\varepsilon}(M)\right),
$$

it follows from Theorem A that

$$
\begin{aligned}
A_{E(\xi)}^{\varepsilon} & =\frac{1}{\varepsilon} v \quad\left(v \text { is a vertical vector of } t_{\varepsilon}(M) \text { at } \xi\right), \\
A_{E(\xi)}^{\varepsilon} \tilde{X}_{\xi} & =\frac{\mu\left(g_{*}^{-1} \xi\right)}{\varepsilon \tan \left(\mu\left(g_{*}^{-1} \xi\right)-\mu(Y)\right)} \tilde{X}_{\xi} \quad\left(X \in\left(D_{\mu}\right)_{x}\right) .
\end{aligned}
$$

Denote by $V^{\varepsilon}$ (respectively $H^{\varepsilon}$ ) the vertical (respectively horizontal) distribution on $t_{\varepsilon}(M)$ and by $\tilde{D}_{\mu}$ the natural lift of $D_{\mu}$. Clearly we have $T t_{\varepsilon}(M)=V^{\varepsilon} \oplus H^{\varepsilon}$ and $H^{\varepsilon}=\oplus_{\mu \in \Delta_{+}} \tilde{D}_{\mu}$. Also, since the normal connection is flat, the distribution $H^{\varepsilon}$ is integrable. Define functions $\lambda_{\mu}\left(\mu \in \triangle_{+}\right)$on $t_{\varepsilon}(M)$ by

$$
\lambda_{\mu}(\xi)=\frac{\mu\left(g_{*}^{-1} \xi\right)}{\varepsilon \tan \left(\mu\left(g_{*}^{-1} \xi\right)-\mu(Y)\right)} \quad\left(\xi \in t_{\varepsilon}(M)\right),
$$

where $g$ is the previous element of $G$ depending on $\pi_{\varepsilon}(\xi)$. It is easy to show that the $\lambda_{\mu}\left(\mu \in \triangle_{+}\right)$are mutually distinct at each point of an open dense subset $W$ of $t_{\varepsilon}(M)$. It is clear that $\lambda_{\mu}\left(\mu \in \triangle_{+}\right)$are distinct from $1 / \varepsilon$ at each point of $t_{\varepsilon}(M)$. Thus $\left.\lambda_{\mu}\right|_{W}$ ( $\mu \in \Delta_{+}$) and $1 / \varepsilon$ are all of the principal curvatures of $W$. Also, the distribution $\left.\tilde{D}_{\mu}\right|_{W}$ (respectively $\left.V^{\varepsilon}\right|_{W}$ ) is the curvature distribution for $\left.\lambda_{\mu}\right|_{W}$ (respectively $1 / \varepsilon$ ). Further, the curvature distributions $\left.\tilde{D}_{\mu}\right|_{W}$ and $\left.V^{\varepsilon}\right|_{W}$ extend to $\left(C^{\infty}\right)$ distributions $\tilde{D}_{\mu}$ and $V^{\varepsilon}$, respectively. Thus the constant tube $t_{\varepsilon}(M)$ has a property similar to that of the curvature netted hypersurface defined in [11]. If the normal vector field $\tilde{\xi}$ is parallel with respect to the normal connection of $M$, then $\mu\left(g_{*}^{-1} \tilde{\xi}_{x}\right)$ is independent of the choice of $x \in M$, where $g$ is the previous element of $G$ depending on $x$. This implies that $\lambda_{\mu}$ is constant along the leaves of $H^{\varepsilon}$. In particular, we have $X \lambda_{\mu}=0$ for all $X \in \tilde{D}_{\mu}$. 


\title{
REFERENCES
}

[1] J. Berndt and L. Vanhecke. Curvature adapted submanifolds. Nihonkai Math. J. 3 (1992), 177-85.

[2] J. Berndt. Homogeneous hypersurfaces in hyperbolic spaces. Math. Z. 229 (1998), 589-600.

[3] S. Carter and A. West. Partial tubes about immersed manifolds. Geom. Dedicata 54 (1995), 145-69.

[4] T. E. Cecil and P. J. Ryan. Focal sets and real hypersurfaces in complex projective space. Trans. Amer. Math. Soc. 269 (1982), 481-99.

[5] T. E. Cecil and P. J. Ryan. Tight and Taut Immersions of Manifolds (Research Notes in Mathematics, 107). Pitman, London, 1985.

[6] B. Y. Chen and S. Ishikawa. On classification of some surfaces of revolution of finite type. Tsukuba J. Math. 17 (1993), 287-98.

[7] A. Gray. Tubes. Addison-Wesley, Redwood City, CA, 1990

[8] S. Helgason. Differential Geometry, Lie Groups, and Symmetric Spaces. Academic Press, New York, 1978.

[9] M. Kimura. Real Hypersurfaces and complex submanifolds in complex projective space. Trans. Amer. Math. Soc. 296 (1986), 137-49.

[10] N. Koike. On tubes of non-constant radius. Tsukuba J. Math. 17 (1993), 267-86.

[11] N. Koike. The decomposition of curvature netted hypersurfaces. Geom. Dedicata 54 (1995), 1-11.

[12] J. Milnor. Morse Theory (Ann. Math. Stud., 51). Princeton University Press, Princeton, 1963.

[13] T. Nagano. The involutions of compact symmetric spaces II. Tokyo J. Math. 15 (1992), 39-82.

[14] H. Reckziegel. On the eigenvalues of the shape operator of an isometric immersion into a space of constant curvature. Math. Ann. 243 (1979), 71-82.

[15] M. Takeuchi. On conjugate loci and cut loci of compact symmetric spaces II. Tsukuba J. Math. 3 (1979), 1-29.

[16] C. L. Terng and G. Thorbergsson. Submanifold geometry in symmetric spaces. J. Diff. Geom. 42 (1995), 665-718.

[17] M. Vernon. Some families of isoparametric hypersurfaces and rigidity in a complex hyperbolic space. Trans. Amer. Math. Soc. 312 (1989), 237-56.

[18] M. Vernon. Tubes in complex space forms. Tensor, N. S. 57 (1996), 38-43.

[19] K. Yano and M. Kon. Structures on Manifolds (Series in Pure Mathematics, 3). World Scientific, Singapore, 1984

\author{
Naoyuki Koike \\ Department of Mathematics \\ Faculty of Science \\ Science University of Tokyo \\ Wakamiya-cho 26 \\ Shinjuku-ku \\ Tokyo 162-0827 \\ Japan
}

(E-mail:koike@ma.kagu.sut.ac.jp) 